\title{
Compact Objects and Accretion Disks
}

\section{By ROGER BLANDFORD ${ }^{1}$, ERIC A G OL ${ }^{1}$, A VERY BRODERICK ${ }^{1}$, JEREMY HEYL ${ }^{2}$, LEON KOOPMA N S ${ }^{1}$, HEE-W O N LE E}

\author{
${ }^{1}$ Theoretical Astrophysics, Caltech, Pasadena, CA 91125, USA \\ ${ }^{2}$ Center for Astrophysics, 60 Garden St., Cambridge, MA 02173, USA \\ ${ }^{3}$ Yonsei University, Seoul, Korea
}

\begin{abstract}
Recent developments in the spectropolarimetric study of compact objects, specifically black holes (stellar and massive) and neutron stars are reviewed. The lectures are organized around five topics: disks, jets, outflows, neutron stars and black holes. They emphasize physical mechanisms and are intended to bridge the gap between the fundamentals of polarimetry and the phenomenology of observed cosmic sources of polarized radiation, as covered by the other lecturers. There has been considerable recent progress in spectropolarimetry from radio through optical frequencies and this is producing some unique diagnostics of the physical conditions around compact objects. It is argued that there is a great need to develop a correspondingly sensitive polarimetric capability at ultraviolet through $\gamma$-ray energies.
\end{abstract}

Spectropolarimetric observations, particularly those at radio and optical wavelengths, have played an important role in high energy astrophysics. From the discovery of synchrotron radiation to the first good evidence for AGN unification, from the polarization patterns in the coherent emission of radio pulsars to the discovery of variable, linear polarization in the absorption troughs of broad absorption line quasars, polarization studies often provide the best and sometimes the only clue we have as to the geometric disposition of the emitting elements in these diverse sources when we cannot resolve them directly.

These notes summarize lectures delivered by Roger Blandford at the XII Canary Islands Winter School on Astrophysical Spectropolarimetry. They are written up with the assistance of Eric Agol (Disks), Leon Koopmans (Jets), Hee-Won Lee (Outflows), Jeremy Heyl (Neutron Stars) and Avery Broderick (Black Holes) The lectures were intended to provide a bridge between the general physical foundations of polarimetry and its practical description presented at the school by Drs. Landi Degl'Innocenti and Keller and the observationally oriented lectures of Drs. Antonucci and Hildebrand. They also make some important connections to solar, stellar (especially white dwarf) and maser polarimetry as described by Drs. Stenflo, Mathys and Elitzur, respectively. They are organised around five generic astrophysical sources: disks, jets, winds, neutron stars, and black holes. In each case a cursory motivation is provided by summarizing some relevant observations and presenting some of the key issues that polarimetry can help to resolve. This is followed by an heuristic discussion of some relevant physical mechanisms in a manner which, it is hoped, will allow them to be applied elsewhere followed by a brief account of how they have been used so far and some suggestions of possible future investigations.

\section{Disks}

\subsection{Motivation}

Accretion disks are commonly found when gas, with angular momentum, is gravitationally attracted towards a central massive body (Frank, King, \& Raine 1992). First 
described in the context of Laplace's nebular hypothesis and first seriously analyzed by Lüst (1952) they have been observed around black holes, neutron star and white dwarf binaries (Shapiro \& Teukolsky 1983), around protostars, and especially within active galactic nuclei, including quasars (Blandford, Netzer, \& Woltjer 1990, Krolik 1999). It is this last type of disk that provides us with much of our most detailed observations. Although it is not part of my task to discuss them, observations of young stellar objects and cataclysmic variables are turning out to be particularly instructive and much of what follows is informed by the results of these studies.

Disks are planar structures and if their opacity is predominantly scattering, by either free electrons or dust grains, then the direction and strength of the polarization tells us about the orientation and inclination of the disk as well as the location of the continuum source. As we shall discuss, (cf also Antonucci, Hildebrand, these proceedings), most astrophysical disks are associated with jets or bipolar outflows and when these can be resolved, they may represent the projected rotation axis of the inner disk. (As we shall also see, disks can be warped and this axis can change with radius and, in the case of AGN, it may be quite different from the axis of the host galaxy.)

We wish to use polarization observations to determine what disks are really like. Unfortunately, the current observational capability is limited. Polarimetry in the radio, the near infrared and the optical regions of the spectrum is really quite good by astronomical, (although not solar), standards. Optically, spectropolarimetry has been performed at the 0.001 level down to $R \sim 18$. Measurement in the mid and far infrared is more of a challenge, though one that has been met in the far infrared, (Hildebrand, these proceedings).

However, to understand the inner disk we need ultraviolet and X-ray polarimetry. The former was carried out for a while on bright quasars using the HST Faint Object Camera, as we shall describe in section 3 below, although this has proved to be a little controversial. $\mathrm{X}$-ray polarimetry has really only been accomplished successfully on a few bright sources (Mészarós et al.1988). There are plans to fly a more sensitive polarimeter on Spectrum-X. It will become clear that there is a very strong scientific case to be made to develop X-ray polarimetry. There is also a strong incentive to develop a $\gamma$-ray polarimetric capability though, here, the technical challenges are even greater. In principle, Compton telescopes operating at $\sim \mathrm{MeV}$ energies, record polarimetric information though, in practice, it has proven to be almost impossible to extract this signal from observations taken to date.

\subsection{Observation}

A particularly good example of an AGN accretion disk can be found in NGC 4258 (cf Elitzur, these proceedings). Here water maser observations reveal a resolved, disk orbiting a 43 million solar mass black hole (beyond all reasonable doubt). We have believed for a long while that gas moves radially inwards through this disk as a result of a hydromagnetic torque that transports angular momentum outward. The binding energy that is released by the infalling gas can be radiated away and this process accounts for the most luminous of quasars and binary X-ray sources. It can also be responsible for driving powerful outflows, as we shall see. Evidence that disks can extend all the way down to the central compact object has been provided by ASCA X-ray observations of relativistically-broadened fluorescent iron emission lines from low luminosity Seyfert and LINER galaxies. (This interpretation has been somewhat controversial; observations, with superior sensitivity and spectral resolution, from XMM-Newton are therefore eagerly awaited.)

Not all disks are thin. There are good phenomenological reasons to suspect that the disks contained in many Seyfert and LINER galaxies thicken over some radii to form 


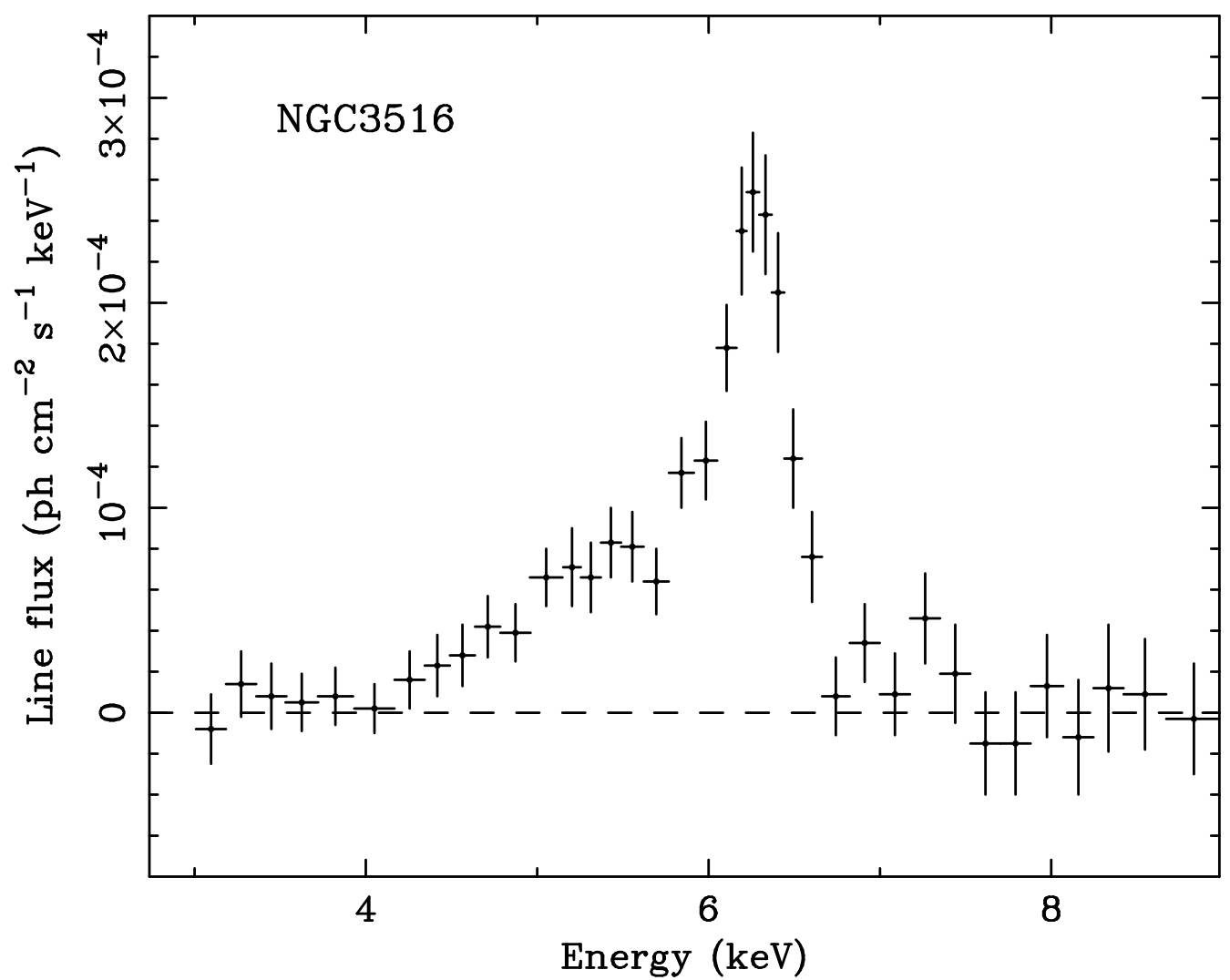

Figure 1. Broad Fe K $\alpha$ line from NGC 3516 observed with ASCA by Nandra et al.(1999).

dense, obscuring torii. Theoretically, it has been proposed that the inner regions of disks that are supplied with gas at a rate that is either much higher or much lower than the Eddington rate (given by $\dot{M}_{\mathrm{Edd}}=L_{\mathrm{Edd}} / c^{2}=4 \pi G M / c \kappa$ ) will thicken because the gas will be unable to cool and the inflow may even become quasi-spherical. Similarly, the disk in NGC 4258 is clearly warped and this is thought to be a common occurrence. It has even been proposed that radiative torques acting on disks can turn them over (Pringle 1997). Polarization observations offer the opportunity to probe the complex geometry of these flows.

Another example of a subtle arrangement of the emitting elements is provided by Xray observations of the thin disks in Seyfert galaxies. The current working model is that only a fraction of the binding energy released by the accreting gas is radiated from the disk photosphere (with an effective temperature in the $\mathrm{keV}$ range). The remainder is dissipated in a hot corona, presumably as a consequence of magnetic flaring followed by reconnection and hydromagnetic wave damping. The heated electrons can then scatter the escaping soft photons and, as the corona is probably Thomson thick, this will lead to a power-law tail in the hard X-ray spectrum. However, roughly half of these photons will strike the disk where they can be absorbed if they have low energy and suffer inelastic Compton recoil loss at high energy. The reflected spectrum will therefore be convex and be imprinted with line features, most famously, the $6.4 \mathrm{keV} K \alpha$ line of Fe (Fabian et al.2000 and references therein, Fig. (1)). On this basis, it has been argued that the widths of lines in Seyfert and LINER galaxies like MCG 6-30-15 imply that the central black hole is rapidly spinning. However, the details depend upon the relative location of 
the region where the Comptonisation is taking place and the region of the disk responsible for most of the reflection. There are two ways to test these models using more detailed observations. The first is to use a technique called reverberation mapping (e.g. Young \& Reynolds 2000). This requires monitoring the variation of the line and the continuum simultaneously and deriving the lag in the variation of the former in response to the latter. This tells us about the geometry and size of the line-emitting region. The second technique is to use polarization observations which will test the geometry.

\subsection{Physical Processes}

We now summarize some relevant physical processes. A good general reference is Rybicki \& Lightman (1979).

\subsubsection{Thomson Scattering}

Classical Thomson scattering is strongly polarizing. A free electron can be considered as a Larmor dipole driven by the electric field of the incident wave (with polarization vector $\vec{e}$ ). The scattered power into polarization is $\vec{e}$ is given by

$$
\frac{d \sigma}{d \Omega^{\prime}}=r_{e}^{2}\left(\vec{e} \cdot \vec{e}^{\prime}\right)^{2}
$$

where $r_{e}=e^{2} / m_{e} c^{2}=2.82 \times 10^{-13} \mathrm{~cm}$ is the classical electron radius. Note that when the scattering angle is $\phi=90^{\circ}$, the radiation is 100 percent polarized.

Averaging over incident polarization and summing over final polarization states gives the familiar differential cross section

$$
\frac{d \sigma}{d \Omega^{\prime}}=\frac{1}{2} r_{e}^{2}\left(1+\cos ^{2} \phi\right) .
$$

Integrating over solid angle gives the total Thomson cross section

$$
\sigma_{T}=\frac{8 \pi}{3} r_{e}^{2}=6.65 \times 10^{-25} \mathrm{~cm}^{2} .
$$

\subsubsection{Compton scattering}

At X-ray and $\gamma$-ray energy, we must take account of the electron recoil. Conserving energy and linear momentum, we obtain an expression for the scattered energy $\epsilon^{\prime}$ in terms of the incident energy $\epsilon$

$$
\epsilon^{\prime}=\frac{\epsilon}{1+\frac{\epsilon}{m_{e} c^{2}}(1-\cos \phi)} .
$$

Averaging over $\phi$ for small values of $\epsilon$, we obtain the mean energy shift

$$
<\Delta \epsilon=-\frac{\epsilon^{2}}{m_{e} c^{2}} .
$$

The Thomson cross section must be replaced by the Klein-Nishina cross section

$$
\frac{d \sigma}{d \Omega^{\prime}}=\frac{1}{2} r_{e}^{2}\left(\frac{\epsilon^{\prime}}{\epsilon}\right)^{2}\left[\frac{\epsilon^{\prime}}{\epsilon}+\frac{\epsilon}{\epsilon^{\prime}}-\sin ^{2} \phi\right],
$$

which emphasizes forward over backward scattering. High energy scattering is nearly as strongly polarizing as Thomson scattering. Averaging over $\phi$ again for small angle, we obtain

$$
\sigma=\sigma_{T}\left(1-\frac{2 \epsilon}{m_{e} c^{2}}+\ldots\right) .
$$


For a large photon energy, $\epsilon>>m_{e} c^{2}$, we have

$$
\sigma \sim \frac{3 \sigma_{T}}{8 \epsilon}\left[\log \left(\frac{2 \epsilon}{m_{e} c^{2}}\right)+\frac{1}{2}\right]
$$

The rate at which the photons heat the electrons through Compton recoil is therefore given for $\epsilon<<m_{e} c^{2}$ by

$$
W_{+}=n_{e} \sigma_{T} c \int d \epsilon N(\epsilon) \frac{\epsilon^{2}}{m_{e} c^{2}}=n_{e} \sigma_{T} c U \frac{<\epsilon>}{m_{e} c^{2}},
$$

where $N(\epsilon)$ is the photon number density per unit energy, $U=\int d \epsilon \epsilon N$ is the photon energy density and $<>$ should be interpreted as an energy density-weighted photon energy.

These expressions describe the energy loss in the initial rest frame of the scattering electron. However when the plasma is hot the electron will be moving and this will cause the photon to experience a Doppler shift. To $O(v / c)$, blue shifts balance redshifts and there is no net energy change when the electron distribution is isotropic. However, it is apparent what there will be a net energy gain to $O(v / c)^{2}=O\left(k T / m_{e} c^{2}\right)$, because the rate of approaching collisions will exceed the rate of receding collisions. We can therefore express the net rate of energy loss by the electrons in terms due to the Doppler shift as

$$
W_{-}=n_{e} \sigma_{T} c U \frac{x k T}{m_{e} c^{2}},
$$

where $x$ is a number that we can fix by observing that there should be energy balance, $W_{+}=W_{-}$, when we use a dilute black body of temperature $T$

$$
N(\epsilon) \propto \epsilon^{2} \exp [-\epsilon / k T] .
$$

(It is necessary to use a dilute black body to avoid having to consider nonlinear, induced Compton scattering.) As $\langle\epsilon\rangle=4 k T$, we deduce that $x=4$.

If this were the dominant physical process, then the equilibrium electron temperature in a given radiation field would be

$$
T_{c}=\frac{<\epsilon>}{4 k} .
$$

However, accretion disk corona are probably heated through reconnection and hydromagnetic turbulence, and the temperature is probably quite non-uniform and hard to predict in detail. An additional complication is that the thermalization timescales are actually quite long compared with the disk dynamical timescale and so the plasma is likely to have a significant suprathermal component (Gierlinski et al.1999).

More generally, we can deduce the form of the kinetic equation for the photon distribution. As the individual photon energy shifts are small, this will have the form of a modified diffusion equation in energy space. However, as the scattering angles are typically large, we cannot regard this as a diffusion in momentum space. We therefore just consider an isotropic radiation field to bring out some principles (although this approximation is inappropriate for computing polarization, since an isotropic radiation field creates zero net polarization upon scattering). As Compton scattering conserves the number density of photons the equation must have the form

$$
\frac{\partial N}{\partial t}=-\frac{\partial F}{\partial \epsilon}
$$

where $F$ is the flux of photons in energy space. Now for a dilute black body, Eq. (1.11), $F$ will be linear in $N$, as long as we can ignore induced scattering, and, as it represents a diffusion, $F$ will contain the first derivative of $N$ with respect to energy. As $F$ must 
vanish for a dilute black body it must have the form

$$
F(\epsilon)=-g(\epsilon)\left(\frac{\partial\left(N / \epsilon^{2}\right)}{\partial \epsilon}+\frac{N}{\epsilon^{2} k T}\right),
$$

for some function $g(\epsilon)$. We next multiply this equation by $\epsilon$ and integrate over energy and use either Eq. (1.9) or Eq. (1.10) to identify the function $g(\epsilon)=n_{e} \sigma_{T} c \epsilon^{4} k T / m_{e} c^{2}$. The resulting (Fokker-Planck) equation is,

$$
\frac{\partial N}{\partial t}=\frac{n_{e} \sigma_{T}}{m_{e} c} \frac{\partial}{\partial \epsilon} \epsilon^{2}\left[\epsilon^{2} k T\left(\frac{\partial\left(N / \epsilon^{2}\right)}{\partial \epsilon}\right)+N\right] .
$$

This formalism describes the behavior of electrons interacting with a dilute gas of photons inside a box with reflecting walls. If we need to take account of induced scattering processes, then Eq. (1.14) must be modified so $F=0$ when $N(\epsilon)$ has the Planck form,

$$
N(\epsilon)=\frac{8 \pi \epsilon^{2}}{h^{3} c^{3}}[\exp (\epsilon / k T)-1]^{-1} \text {. }
$$

The result is that $N \rightarrow N(1+N)$, in the second term in brackets in Eq. (1.15) which is then known as the Kompaneets equation and is central to discussions of the transfer of radiation through hot plasmas.

Clearly the radiation as described by this isotropic formalism will be unpolarized. In order to describe the polarization of a cosmic source, we must tackle the radiative transfer. There are three approaches that have commonly been followed.

(a) Escape Probability Formalism This is the simplest approach. We add a term to the right hand side of Eq. (1.15)

$$
-\frac{N c}{R(1+\tau)} .
$$

The extra factor $1+\tau$ takes into account the impeded photon escape when the Thomson depth $\tau$ exceeds unity. This approach, which is most commonly used, although instructive as far as the spectrum goes, is not much help when it comes to polarization.

(b) Intensity Formalism Provided that we restrict our attention to simple shapes slabs, spheres etc. - we can incorporate the energy space transport within the equation of radiative transfer using a scattering kernel. This can then be solved by taking moments and imposing a closure relation in the standard manner. It is possible to include polarization though this leads to quite involved equations.

(c) Monte Carlo Formalism In many ways the most versatile method is the same one used in nuclear reactors for the transport of neutrons. This is to follow individual photons, within the scattering region starting with energies, locations and directions that are selected according to a prescribed distribution using random numbers. Polarization is relatively easy to handle, and most polarization is created in the last few scatterings, reducing the computational burden which occurs at large optical depths. All of this is quite straightforward in principle, though, in practice, Monte Carlo simulations are quite an art as a variety of ingenious tricks have to be used to reduce the variance with a finite amount of computer time.

\subsubsection{Dust Scattering}

Dust scattering is more complex than Thomson scattering as the cross section depends on wavelength, grain size, and grain composition (cf Hildebrand, these proceedings). In the limit when the wavelength is much greater than the grain size, Rayleigh scattering applies, which has the same angular cross section as Thomson scattering, but scales as $\lambda^{-4}$, a fact which has been used to distinguish electron scattering from dust scattering in 
some Seyfert 2 galaxies. For a range of different sizes and compositions, it is impossible to express the dust scattering cross section in a simple formula, but extensive numerical calculations have been carried out by, e.g., Draine \& Lee (1984), Zubko \& Laor (2000).

\subsubsection{Faraday Rotation}

The next relevant physical process is Faraday rotation. When electromagnetic wave modes propagate through a plasma, their phase velocities will be changed from $c$. To lowest order, the eigenmodes are circularly polarized with phase velocity difference

$$
\Delta V_{\phi}=2 c \frac{\omega_{p}^{2} \omega_{G}}{\omega^{3}} \cos \alpha
$$

where $\omega_{p}=\left(4 \pi n_{e} e^{2} / m_{e}\right)^{1 / 2}$ is the electron plasma frequency, $\omega_{G}$ is the electron gyro frequency and $\alpha$ is the angle between the ray and the magnetostatic field. If we decompose a linear polarized wave into two circularly polarized modes that propagate through the medium and then recombine the modes after they leave the medium, then there will be a net rotation of the plane of polarization through an angle

$$
\frac{d \Phi}{d \tau_{T}}=\frac{\omega}{2} \int d s \frac{\Delta V_{\phi}}{c^{2}}=0.1\left(\frac{\lambda}{500 \mathrm{~nm}}\right)^{2}\left(\frac{B_{\|}}{1 \mathrm{G}}\right)
$$

Polarization observations can tell us as much about the intervening medium as about the source.

\subsubsection{Relativistic Radiative Transfer}

Another interesting complication is that special and general relativistic effects will affect the transfer of radiation from the disk to us. This is particularly interesting for line radiation. The non-relativistic Doppler shift will broaden the profile of a line formed at the photosphere of a rotating disk, with the blue wing coming from the approaching limb and the red wing from the receding limb. The gravitational shift (which is not separated from the Doppler shift in a general relativistic calculation) will accentuate the red wing whose extent depends upon how close the inner edge of the disk gets to the hole. A further effect is that rays will be deflected by the gravitational pull of the central black hole so that an image of an accretion disk observed from near the equatorial plane would exhibit the back side of the disk. (There are ambitious proposals to deploy an $\mathrm{X}$-ray interferometer in space which could exhibit these and other effects.)

Of more direct relevance to this school, is the behavior of the polarization. There are two main effects. Firstly, aberration changes the emission angle, and consequently the emitted polarization, in the rest frame of the orbiting gas. Secondly, the plane of polarization is rotated as the ray propagates near the black hole (Fig. (2)). As we discuss in more detail in $\S 5$, the polarization direction is parallel-propagated along null geodesics. All of this is straightforward, if somewhat tedious, to compute. Specific models for continuum emission have been computed by Laor et al.(1990) and Connors et al.(1980), while relativistic effects on line polarization were computed by Chen \& Eardley (1991). It may be possible to use observations of the rotation of the polarization direction with wavelength, in a spectral line, or in the continuum if the wavelength is a measure of the effective radius of the disk, to measure the spin of the hole.

\subsection{Interpretation}

Having outlined some of the relevant physical mechanisms, let us return to the problem with which we began this section. "How much polarization do we expect from an accretion disk and what do we observe?" We can split the problem into two parts - the escape 


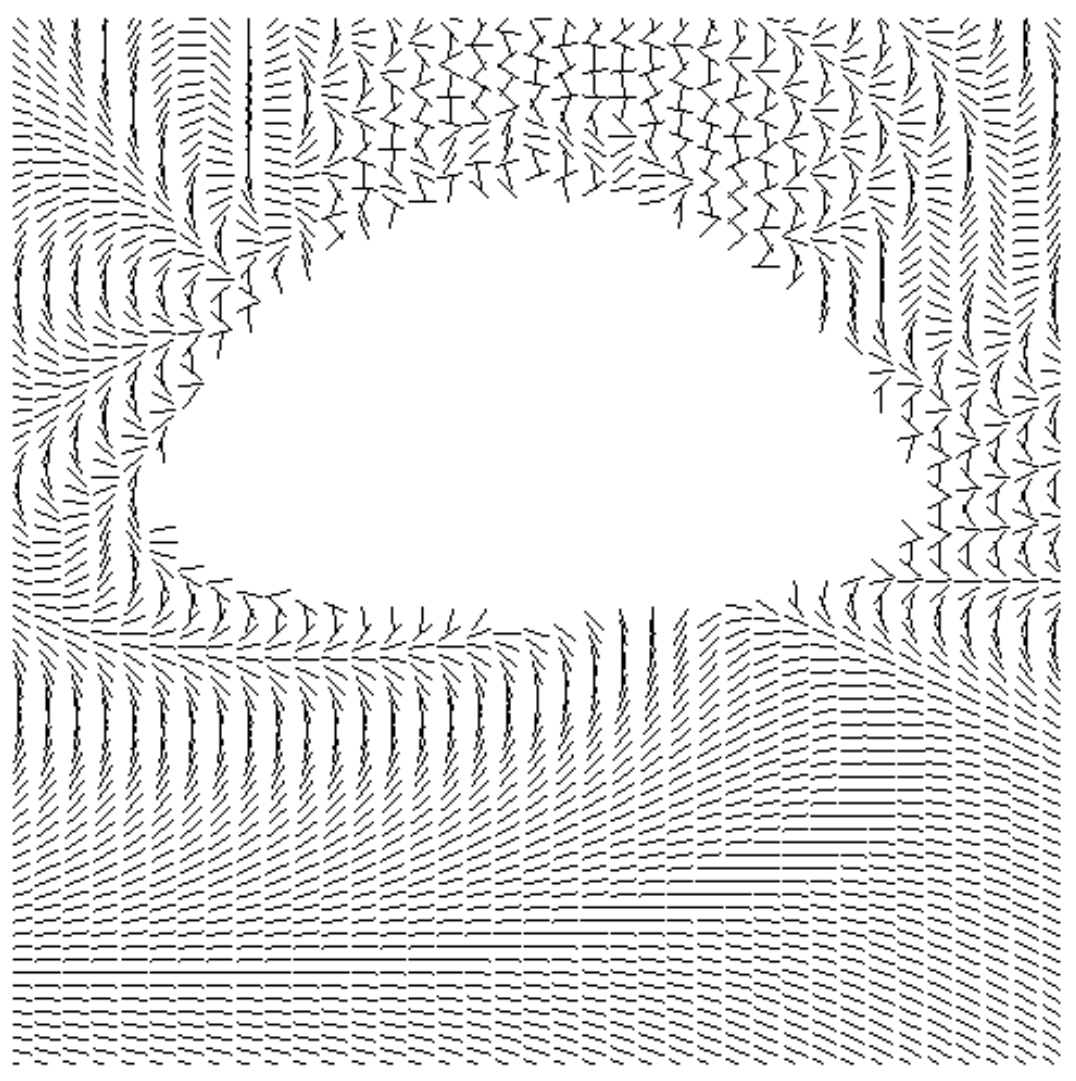

Figure 2. Polarization angle as a function of position for an electron-scattering dominated thin accretion disk around a Kerr black hole $(\mathrm{a}=0.998)$ viewed at infinity from an angle of $75^{\circ}$. The figure is $20 G M / c^{2}$ in size.

of photons emitted in the disk and the behavior of the scattered photons. For a given atmosphere, the problem is linear and we can superpose the two components. However, if we try to solve self-consistently for the ionization and thermal state of the atmosphere, the problem becomes nonlinear. 


\subsubsection{Electron Scattering}

Radiative transfer in a pure scattering, plane parallel atmosphere is a classical problem that was solved for a Thomson scattering kernel, initially analytically, by Chandrasekhar (1960) and then in greater generality by Angel (1969) using a Monte Carlo approach. The answer is that the emergent polarization varies with inclination, having a value $p=0.12$ when the atmosphere is viewed horizontally and $p=0.02$ when viewed at the most probable inclination of $60^{\circ}$ and, of course, $p=0$ when viewed normally. Real disk atmospheres also have an absorptive opacity and this will reduce (or increase) the emergent polarization significantly (Hubeny et al.2000). In addition, we now believe that accretion disks are strongly magnetized. The rationale for this is that ionized accretion disks are known to be unstable to developing strong internal magnetic fields with interior magnetic pressures estimated to be $\sim 1-10$ percent of the gas pressure. This magnetic field will surely be carried out beyond the photosphere and into the coronae (discussed above) by buoyancy forces. Furthermore, magnetic pressure is likely to dominate gas pressure in an accretion disk corona, just like in the solar corona.

When we consider the specific parameters appropriate to observed disks, we find that thermal emission should be unpolarized based on the following argument. Consider a given disk annulus, the radiation pressure at the photosphere, $a T^{4} / 3$, is smaller than the gas pressure within the disk. Because observations at a given thermal wavelength peak near $\lambda \sim h c / k T$, we can derive a lower limit on the magnetic field strength $B \gtrsim 10^{2}(\lambda / 500 \mathrm{~nm})^{-2} \mathrm{G}$. Since photons traverse differing optical depths after their last scattering and the magnetic field will likely have significant inhomogeneities, any polarization will be erased by the Faraday rotation $\left\langle d \Phi / d \tau_{T}\right\rangle \gtrsim 10$, independent of the wavelength observed or the physical size of the disk (Agol \& Blaes 1996).

Thus, it should come as no surprise that AGN disks are generally only polarized by a small amount (Antonucci, this volume). Furthermore, the polarization that is observed may be imprinted extrinsically. Purported rises in polarization below $912 \AA$ in a few quasars observed with HST contradict old theoretical predictions and stand as a challenge to disk theory (Koratkar \& Blaes 1999).

Turning to X-ray wavelengths, where non-thermal emission means that we might ignore Faraday rotation, but cannot ignore reflection, predictions of the emergent polarization under a variety of models have been presented by Matt, Fabian, \& Ross (1993) and Poutanen \& Svensson (1996) and references therein, as shown in Fig. (3). The rather flat $\mathrm{X}$-ray spectrum is created by Compton scattering of thermal emission from the accretion disk, which is partly due to absorbed X-rays. The electron-scattering reflection feature is suppressed at low energies, $\lesssim 8 \mathrm{keV}$, by X-ray bound-free absorption opacity, and at high energies, $\gtrsim 100 \mathrm{keV}$, reduced by electron recoil; consequently, the largest $\mathrm{X}$ ray polarizations should lie between these energies. The magnitude of the polarization may depend upon the exact geometry and placement of the coronal emission regions, an unexplored problem.

\subsubsection{Dusty Disks}

The outer parts of accretion disks may be cool enough $(T<1800 \mathrm{~K})$ to be inhabited by dust grains. If the dusty disk drives a wind, or is inflated or warped, then dust will scatter the light from the inner disk, imprinting a polarization signature from the infrared to ultraviolet. It may in practice be quite difficult to distinguish between a dusty disk, torus, or outflow using polarization, as the level of polarization expected is quite small, $\sim 1 \%$, and depends on the details of the dust model, e.g. Königl \& Kartje (1994). In addition, dust extinction can create polarization if the grains, charged by collisions with ions, are aligned by magnetic fields, inducing polarization at the percent level as well. 


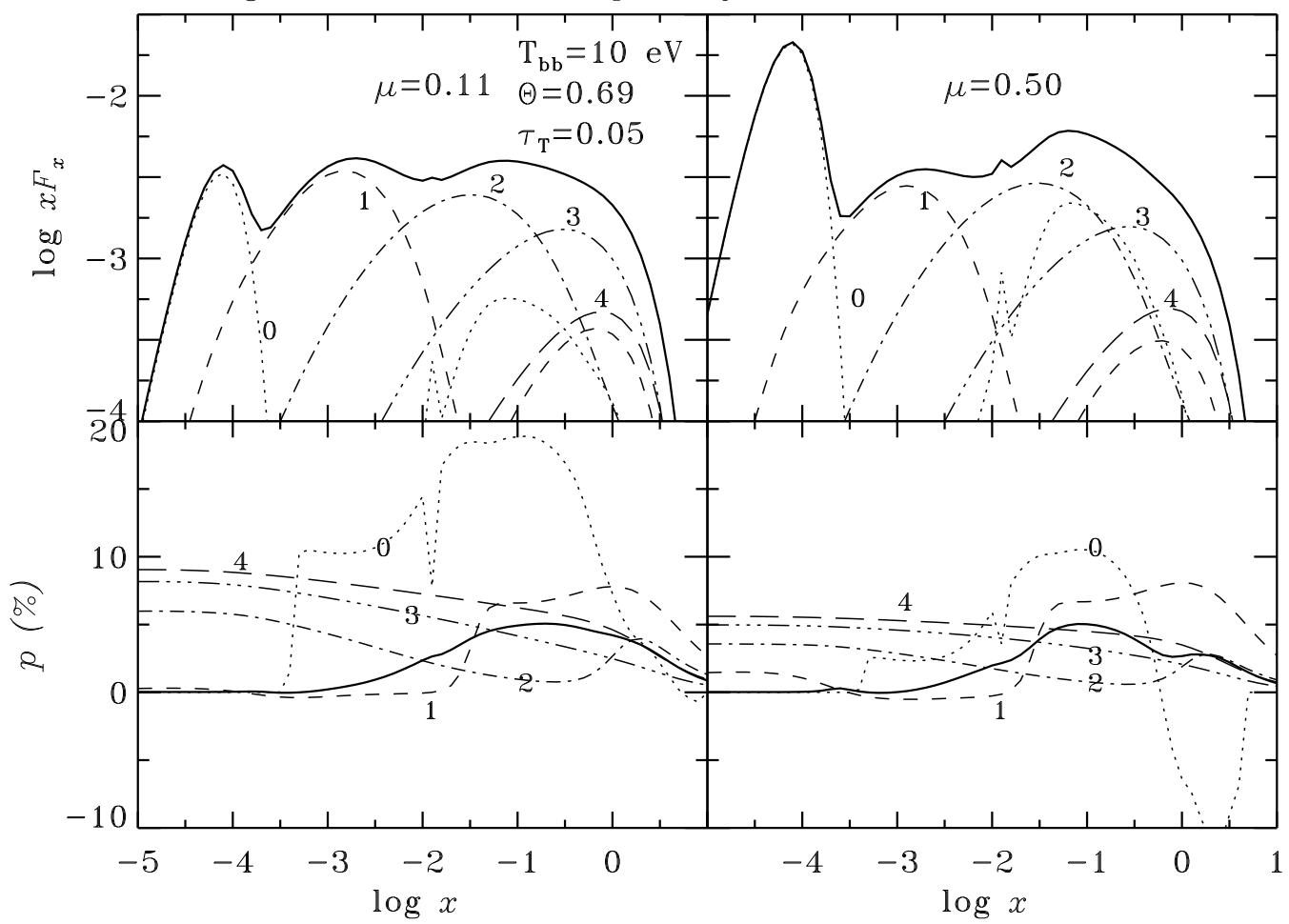

FiguRE 3. Spectrum and polarization versus frequency $\left(x=h \nu / m_{e} c^{2}\right)$ of hot, plane-parallel corona $\left(T_{e}=0.11 m_{e} c^{2}, \tau_{T}=0.05\right)$ above a cold disk viewed at two inclination angles, $\mu=\cos i=0.11$ and 0.5 . The numbers label various scattering orders for reflection, while the solid lines show the total spectrum and polarization (from Poutanen \& Svensson 1996). No relativistic propagation effects have been included.

\subsection{Summary}

- Disks are commonly found in accreting systems.

- Model accretion disks can create strong polarization both in transmission and in reflection, throughout the electromagnetic spectrum.

- Measurement of the variation of linear polarization with wavelength can, in principle, reveal a lot about the disk structure and the location of coronal emission sites.

- However, the situation is, in practice, more complex, particularly at optical wavelengths, where external illumination, warping, and especially Faraday rotation are likely to be very important.

- Even when polarization cannot be measured, its effects are so large under conditions of strong electron scattering that radiative transfer calculations should include polarization.

- Monte Carlo techniques are well-suited for computing polarization in a given model.

- There is a very strong case for developing X-ray polarimetry.

\section{Jets}

\subsection{Motivation}

Jets, or more generally bipolar outflows, are also surprisingly common. They have been studied in association with active galactic nuclei (AGN), binary X-ray sources, young stellar objects, novae and so on. Jet speeds are typically a few times the escape velocity 
from the central object; in the case of black holes, bulk Lorentz factors of $\gamma \sim 10$ are inferred. (Gamma ray bursts may also produce jets with Lorentz factors $\gamma \sim 300$.) Jets are so common that it has been speculated that they may be an essential concomitant of accretion flow - the channels through which the liberated angular momentum and perhaps also much of the energy leave the system. The challenge to the astrophysicist is to explain how jets are powered and collimated. However, even after decades of work, major theoretical and observational questions about their origin, collimation and even their constituents still remain.

There are, generically, two proposed origins for the jet power: the central object (black hole, neutron star or protostar) and the accretion disk (e.g. Blandford et al.1990). In both cases, the energy derives from differential rotation. For example in the case of a Keplerian disk that extends down to the surface of a non-rotating, unmagnetized star, as much energy is released in the boundary layer as in the disk. An extreme case is presented by the Crab (Weisskopf et al.2000) and Vela (Helfand, Gotthelf \& Halpern 2001) pulsars which exhibit prominent jets without there being any accretion disk, presumably.

One of the best observational approaches to investigate the mechanisms which produce jets is to determine the jet composition at radii where they can be observed directly. In the case of black-hole jets, the plasma is likely to be electron-ion if the jet originates from a disk, or electron-positron if it derives from the black hole. Polarization observations have been prominent in attempts to distinguish between these two possibilities. Most contemporary explanations of the collimation invoke strong magnetic fields, though in most cases, the argument for magnetic collimation is a theoretical one, based upon eliminating the alternatives. One exception to this is the bipolar outflow associated with young stellar objects (YSOs) where polarization observations strongly support the notion that the magnetic field is dynamically important (Akeson \& Carlstrom 1999).

\subsection{Observations}

The first jet observed was that in the nucleus of the elliptical galaxy M87 in the Virgo cluster. The modern representation is given in Fig. (4) (Perlman et al.1999). In this case, the jet emerges from no more than 100 times the gravitational radius of the central hole $\left(M=3 \times 10^{9} \mathrm{M}_{\odot} ; G M / c^{2}=4 \times 10^{14} \mathrm{~cm}\right)$ and propagates radially outward for a distance $\sim 3 \times 10^{22} \mathrm{~cm}$, seven orders of magnitude larger. The jet, however, is neither homogeneous nor smooth. Superimposed upon an overall decrease in surface brightness as the jets expand away from the central hole are strong side to side variations and bright features. These may reflect a time-dependence at the jet origin or independent, local instabilities. At small radii, the M87 jet is strongly one-sided, and this is generally attributed to Doppler beaming and adduced as evidence for relativistic outflow.

Another good example of an extragalactic jet is Pictor A, which has recently been observed at X-ray energies using Chandra (Fig. (5)); Wilson, Young \& Shopbell, 2001). It shows strikingly efficient collimation and powerful emission from the western hot spot, which is also prominent at optical and radio wavelengths. The Galactic source GRS $1915+105$ shows mild superluminal motion which is interpreted in terms of moving features, probably internal shocks, with space velocities $\sim 0.9 c$ (e.g. Mirabel \& Rodriguez 1994). This is mild compared with most extragalactic compact jets where the speeds are more typically $\sim 0.99 c$, corresponding to bulk Lorentz factors $\gamma \sim 7$. Indeed, there are observational indications that much larger bulk Lorentz factors are produced in extragalactic jets. Independent evidence that these jets are relativistic comes from $\gamma$-ray observations, principally done with the EGRET detector on Compton Gamma Ray Observatory. These showed that those jets that are beamed towards us, and which are collectively known as blazars, are often powerful $\gamma$-ray sources. The total electromag- 


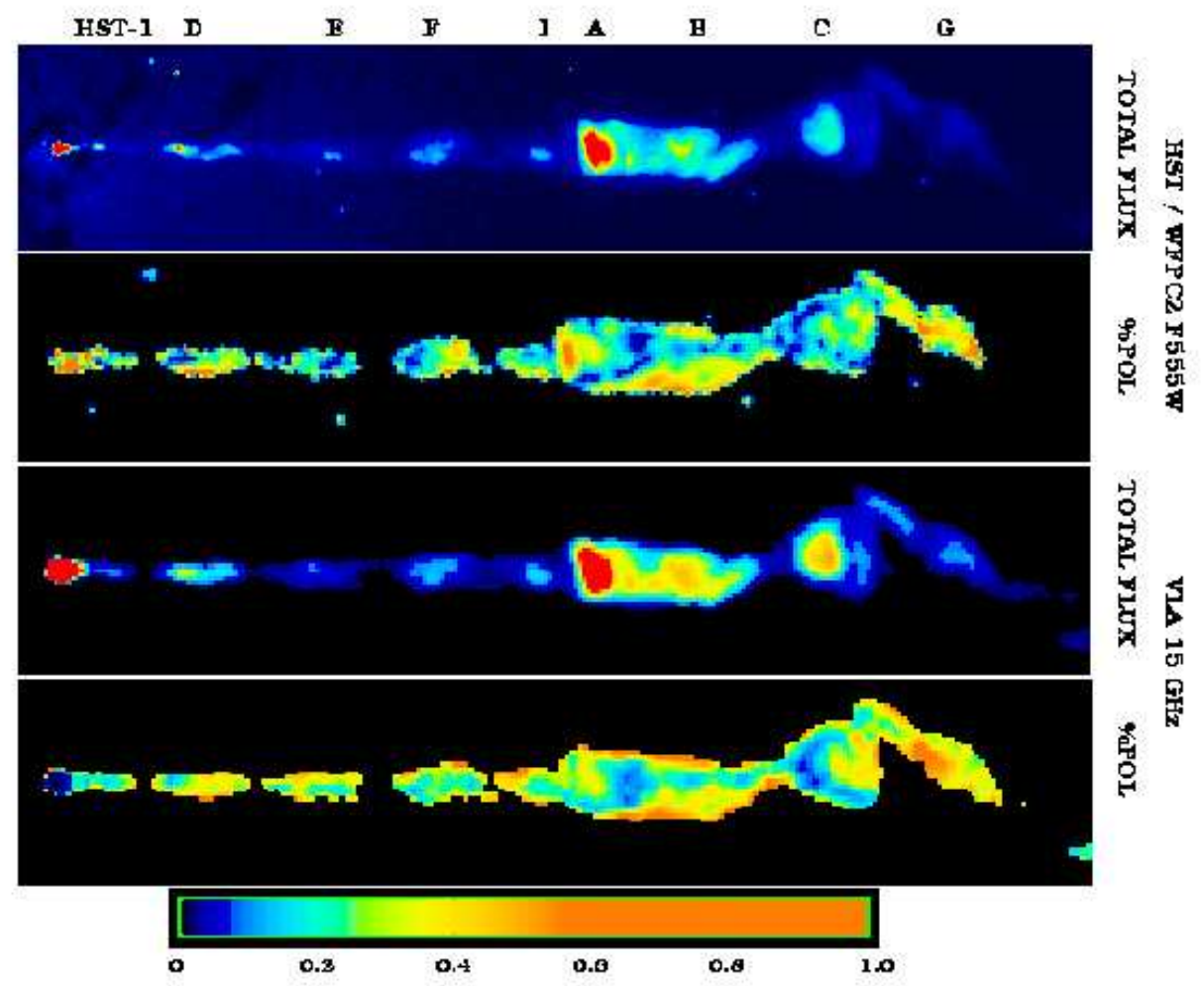

FigurE 4. False-color representations of the total intensity and polarization of the M87 Jet in the optical (HST F555W, top two panels) and radio (VLA $14.5 \mathrm{GHz}$, bottom two panels). The HST observations were carried out in May 1995, while the VLA observations were done in February 1994. All maps were rotated so that the jet is along the x-axis, and are convolved to $0.23^{\prime \prime}$ resolution (from Perlman et al. 1999).

netic spectrum of blazars (and similar sources) comprises a broad band synchrotron radiation spectrum extending from low radio frequencies to an upper frequency between optical and X-ray wavelengths. The same electrons are responsible for an inverse Comp- 


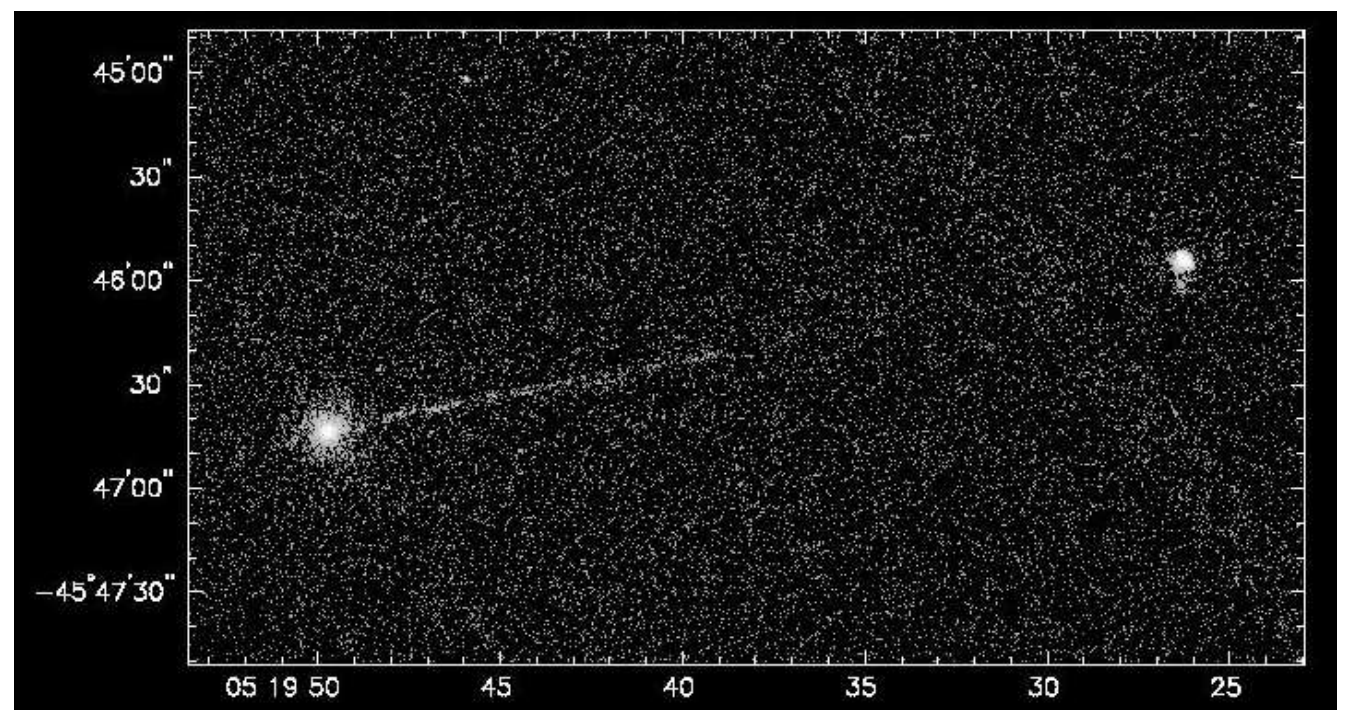

Figure 5. Gray-scale representation of the full-resolution Chandra image of the nucleus, jet and western hot-spot of Pictor A (from Wilson et al.2001).

ton component that can extend up to $\mathrm{TeV}$ energies (with variability times as short as $\sim 30 \mathrm{~min}$ ). (We can only observe $\mathrm{TeV}$ emission from relatively local sources because $\mathrm{TeV}$ photons from cosmologically distant sources will be absorbed through pair production on the intergalactic infrared background.) The fastest jets may well be associated with $\gamma$-ray bursts, if they are indeed beamed, for which speeds of $\sim 0.999995 c$ have been inferred.

\subsection{Physical Processes}

\subsubsection{Synchrotron Radiation}

As described in Dr. Landi Degli'Innocenti's contribution (for more details see also Rybicki \& Lightman 1979), the synchrotron power radiated by an ultra-relativistic electron with energy $\gamma m_{e} c^{2}$ in a field of strength $B$ is given by

$$
P=\frac{4}{3} \gamma^{2} \sigma_{T} c U_{B}
$$

where $U_{B}=B^{2} / 8 \pi$ is the magnetic energy density and we have averaged over pitch angle. The corresponding radiative cooling time is

$$
t_{S}=\frac{5 \times 10^{8}}{B^{2} \gamma} \mathrm{s},
$$

where $B$ is measured in Gauss. The characteristic frequency radiated is

$$
\nu_{c}=\gamma^{2} B \mathrm{MHz} .
$$

The polarization of single particle emission varies from $2 / 3$ for $\nu \ll \nu_{c}$ to 1 for $\nu \gg \nu_{c}$. The electric vector is perpendicular to the projected magnetic field direction. Averaging over a power law distribution of relativistic electrons, $d N / d \gamma=K \gamma^{-s}$, it can be easily shown that the observed intensity is

$$
I_{\nu} \propto K B^{1+\alpha} \nu^{-\alpha}
$$

where the spectral index $\alpha=(s-1) / 2$. The net degree of linear polarization is

$$
\frac{Q}{I}=\frac{s+1}{s+7 / 3} \text {. }
$$


Synchrotron radiation is also naturally circular polarized to an extent

$$
\frac{V}{I} \sim 3 / \gamma,
$$

dependent upon the detailed angular distribution function and the viewing angle.

An important issue for what follows is the viability of a synchrotron maser. This can be shown to be impossible for ultra-relativistic emission in vacuo. Essentially, in order for a maser to operate, it is necessary that there be a population inversion and that the emissivity at a given frequency decreases sufficiently rapidly with increasing energy and this does not happen with regular synchrotron emission. (It can however arise when relativistic electrons emit synchrotron radiation in a plasma, although in practice the conditions for this to occur are rather restrictive. This is known as the Razin effect.)

Of more relevance is what happens when the electrons are no more than mildly relativistic. The emission is then confined to a series of harmonics of the fundamental gyro frequency $\omega_{G} / \gamma$. As the central frequency of a harmonic decreases with increasing energy, there are frequencies and directions where maser action is possible, given a population inversion. Cyclotron masers are likely to be highly polarized. The polarization from an electron-ion plasma will be elliptical; that from an equal pair plasma will be purely linear.

When the brightness temperature $T_{B}=I_{\nu} c^{2} / 2 k \nu^{2}$ of a synchrotron source approaches the kinetic temperature of the emitting electrons, $T_{\mathrm{k}} \sim \gamma m_{e} c^{2} / 3 k \propto(\nu / B)^{1 / 2}$, the radiation will be absorbed. The optically thick radiation from a source will have a brightness temperature that is limited to this value and so the optically thick equivalent of Eq. (2.23) is

$$
I_{\nu} \propto \nu^{5 / 2} B^{-1 / 2} .
$$

When we consider the linear polarization of a self-absorbed source, we observe that the electrons emitting in the field-perpendicular polarization at a given frequency will have slightly lower energies than those emitting in the field-parallel polarization. Therefore the brightness temperature of the field parallel emission will be slightly larger than that of the field perpendicular emission. The degree of linear polarization from a power-law relativistic electron distribution function can be computed to be

$$
\frac{Q}{I}=\frac{-3}{6 s+13} .
$$

An example is the supermassive black hole candidate in the Galactic center, Sagittarius $\mathrm{A}^{*}$, which has no linear polarization up to frequencies of $86 \mathrm{GHz}$ (cf Hildebrand, these proceedings), but shows surprisingly strong circular polarization (e.g. Bower 2000). The degree of $\mathrm{CP}$ increases sharply with frequency. Whereas both advection-dominatedaccretion-flow (ADAF) models and accretion-disk-powered-jet models can account for the spectrum from centimeter wavelengths to X-rays of Sagittarius $\mathrm{A}^{*}$, they have distinct polarization characteristics which might in the near future be able to distinguish between the two models.

\subsubsection{Inverse Compton Scattering}

Inverse Compton (or more properly Thomson) scattering, in which a highly energetic particle transfers momentum to a low-energy photon, is very similar to synchrotron radiation. If the radiation field is isotropic, then the power is given by Eq. (2.20) with the magnetic energy density $U_{B}$ replaced by the radiation energy density, $U_{\text {rad. }}$. As photons are conserved in Thomson scattering, the mean photon frequency is boosted by 
an average factor

$$
\frac{\nu^{\prime}}{\nu}=\frac{4}{3} \gamma^{2}
$$

where $\nu^{\prime}$ is the scattered frequency and $\nu$ is the incident frequency. One power of $\gamma$ arises from the Lorentz transformation into the electron rest frame; the second comes from the scattering back into the original frame. The polarization observed will be generically be $\sim 1 / \gamma$ unless the incident radiation field is both highly anisotropic and polarized, in which case a strongly polarized scattered spectrum can be emitted. This can arise when, for example, radiation is scattered into a beam by a warped disk. Similarly, circular polarization in the incident radiation will be partly retained in the scattered radiation.

\subsubsection{Inverse Compton Limit}

The comparison of synchrotron radiation and inverse Compton scattering leads to what, for historical reasons, is called the inverse Compton limit (Kellermann \& Pauliny-

Toth 1969). The way the argument is traditionally expressed is that the ratio of the Compton power radiated by an electron to the synchrotron power can be written as

$$
\frac{L_{C^{-1}}}{L_{S}}=\frac{U_{S}}{U_{B}} \propto \frac{\nu_{S}^{3} T_{B}}{B^{2}} \propto T^{5} \nu_{S},
$$

in obvious notation, and where we have assumed that the source is self-absorbed at the observing frequency. If we set $\nu_{S} \sim 1-10 \mathrm{GHz}$, then the brightness temperature is limited to $T_{B} \sim 2 \times 10^{12} \mathrm{~K}$ if this ratio is not to exceed unity.

The original concern was that if the ratio did exceed unity then the second order Compton scattering would be even greater than the first order scattering and so on. Of course this can't go on for too many orders because the Klein-Nishina limit will limit the scattering. Nowadays we think we can identify the synchrotron and the inverse Compton components and so we know their ratio and can deduce the source brightness temperature which is quite insensitive to its value.

\subsubsection{Kinematics of Bulk Relativistic Motion}

In the case of a relativistic jet, it is often easier to compute the radiation spectrum in the comoving (primed) frame of the emitting plasma and then perform a Lorentz boost into the (unprimed) frame of the observer. The frequency will be boosted by the Doppler factor (e.g. Blandford \& Königl 1979),

$$
\delta=\frac{\nu^{\prime}}{\nu}=\frac{1}{\gamma(1-\beta \cos \theta)},
$$

where $\theta$ is the scattering angle in the observer frame and $\beta=v / c$ is the bulk velocity of the plasma in the jet. Note that for a jet beamed toward us with $\theta<\gamma^{-1}$, the Doppler factor is $\delta \sim \gamma$. Note also that the rate of change of observer time $t_{\text {obs }}$ to proper time $\tau$ satisfies

$$
\frac{d \tau}{d t_{\mathrm{obs}}}=\delta
$$

As a consequence, the observed transverse speed of a feature moving with the jet speed is given by

$$
\beta_{\mathrm{obs}}=\gamma \beta \sin \theta \frac{d \tau}{d t_{\mathrm{obs}}}=\frac{\beta \sin \theta}{1-\beta \cos \theta} .
$$

This has a maximum value $\gamma \beta$ for $\theta=\cos ^{-1} \beta$ and consequently the expansion can be "superluminal" when $\beta>0.71$. The kinematics of real jets is undoubtedly more complex and the space motion of shock features must be distinguished from the speed of 


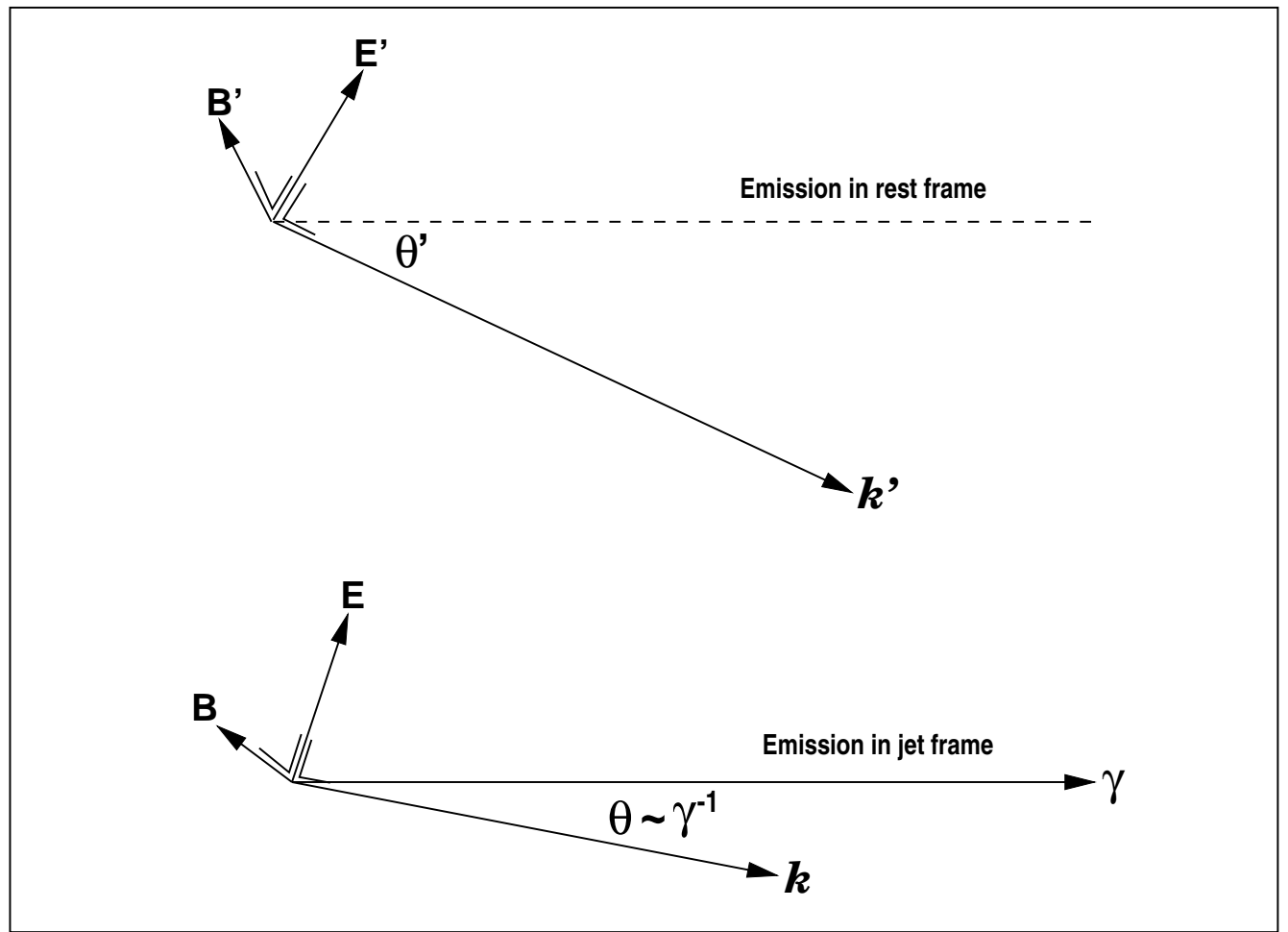

Figure 6. The Lorentz boost of polarization.

the emitting plasma. Frequently, observers make the approximate identification $\beta_{\text {obs }} \sim$ $\theta^{-1} \sim \delta \sim\left(d t / d t_{\text {obs }}\right) \sim \gamma$ when interpreting measurements of compact extragalactic radio sources.

The behavior of polarization under a Lorentz boost is straightforward. The k-vector swings forward along the direction of motion making an angle $\theta^{\prime}$ with the direction of motion in the plasma rest frame and an angle $\theta$ in the jet frame, where $\sin \theta^{\prime}=\delta \sin \theta$. If we imagine the k-vector as being rotated in this manner, then the electric and magnetic fields associated with individual photons will be similarly rotated about a direction $\vec{k} \times \vec{B}$ so that $\vec{k}, \vec{E}$ and $\vec{B}$ continue to form an orthogonal triad (Fig. (6)).

Because the Planck distribution function is Lorentz scalar, and the brightness temperature enters only in the ratio $\nu / T$, it is clear that the brightness temperature must transform in the same manner as frequency. This implies that if the inverse Compton limit is applied in the observer frame, the brightness temperature measured by a radio astronomer can be as high as $\sim 2 \times 10^{12} \delta \mathrm{K}$. This is particularly germane at this time because radio astronomers are able to estimate these brightness temperatures, both directly using ground and orbiting VLBI, and indirectly by carefully analyzing refractive interstellar scintillation. Using the limits on the source size derived from these observations and its relation to the source flux-density (i.e. the Rayleigh-Jeans equation), one can derive an apparent surface brightness temperature and therefore the value of $\delta$, assuming the intrinsic brightness temperature cannot exceed the inverse Compton limit significantly. The values that are found require bulk Lorentz factors $\gamma \sim \delta \sim 30$ and may be even higher. 


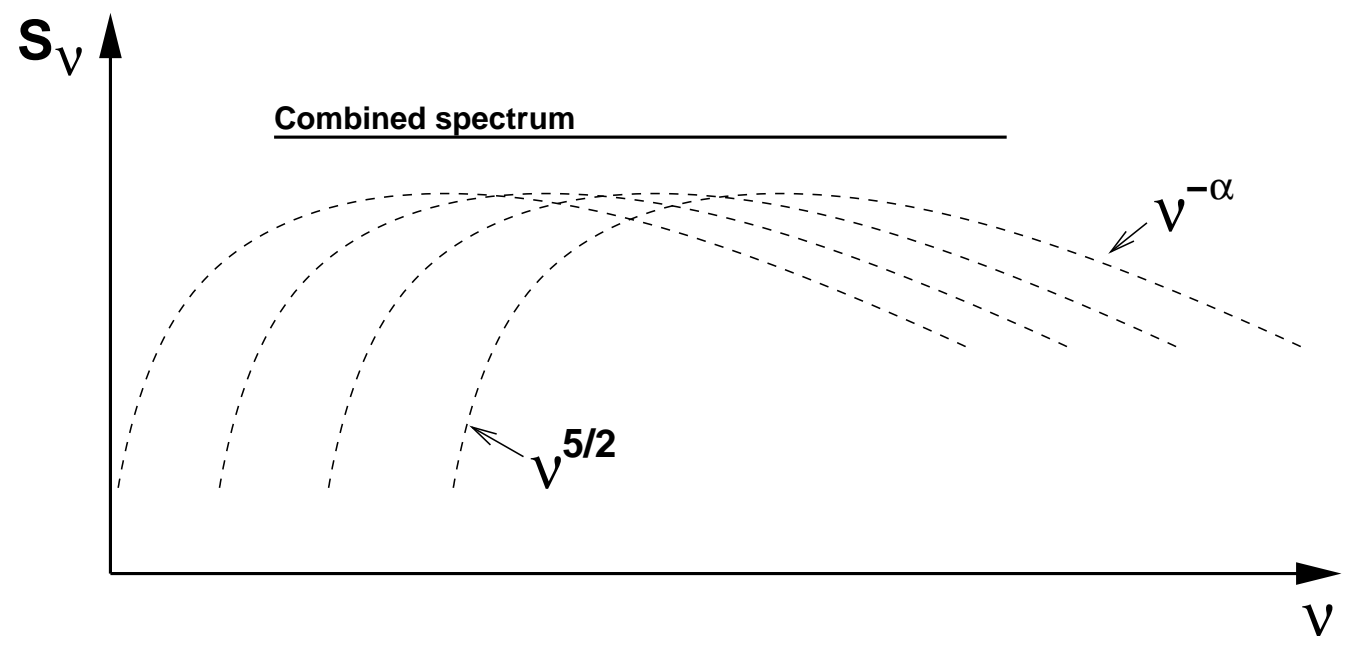

FIGURE 7. A superposition of self-absorbed sources peaking at different frequencies can create a combined 'flat' spectrum.

\subsubsection{Faraday Conversion}

We discussed Faraday rotation in a cold, non-relativistic plasma in the last section. We must now consider what happens in an ultra-relativistic pair plasma. On symmetry grounds, the eigenmodes must be linearly polarized and are usually labeled ordinary, where the electric vector along the direction $\vec{k} \times \vec{B}$, and extraordinary, where it is not. If a linearly polarized wave, obliquely polarized with respect to the magnetic field is incident upon the plasma, then it can be decomposed into ordinary and extraordinary modes that will propagate with slightly different phase velocities. In this manner, circular polarization will be created. In other words, there is a conversion of $U$ to $V$. The sense of circular polarization will be given by the sign of $(\vec{e} \cdot \vec{B})(\vec{e} \cdot \vec{k} \times \vec{B})$ and so in order to have a measurable circular polarization from a cosmic source, it is necessary to have a preferred field orientation. This phenomenon is known as Faraday conversion (e.g. Jones \& O'Dell 1977).

Because Faraday conversion is caused by the lowest energy relativistic electrons, it can serve as a probe of the low energy end of the electron energy distribution. Faraday conversion is furthermore proportional to $e^{2} B^{2} / m^{2}$, i.e. independent from the sign of the particle's charge. An equal mixture of electrons and positrons can therefore produce Faraday conversion, but not rotation. A comparison of linear and circular polarization might therefore probe the constituents of the jet (e.g. electron-positron versus electronion pairs).

\subsection{Interpretation}

\subsubsection{Shocks and the Integrated Spectrum}

The emitting element in powerful synchrotron jets is thought to be a relativistic shock wave and a typical source will comprise the emission from several of them. In the limit, a jet can be thought of as accelerating relativistic electrons over its length with a field strength that diminishes with radius. The total flux density observed at a given frequency is dominated by the emission from the radio photosphere, where the optical depth is roughly unity. Blazars and similar sources generally have "flat" spectra, i.e. $-0.5<\alpha<$ 0.5 and can be interpreted as the superposition of a series of self-absorbed sources at successive radii, each peaking at successively lower frequencies (Fig. (7)). 


\subsubsection{Jet Composition}

On the basis of the fraction of the jet energy per relativistic electron responsible for the synchrotron radio emission that we observe to be radiated on an outflow timescale, it has been tentatively deduced that jets cannot carry protonic "baggage" and so must comprise electron-positron pairs with a low energy cutoff in the distribution function (e.g. Reynolds et al.1996).

Wardle et al.(1998) measure a large degree of circular polarization in $3 \mathrm{C} 279$, a source where the linear polarization is also quite high, and so there cannot be too much normal Faraday rotation. In order to explain the circular polarization, they have to invoke a large population of mildly relativistic electrons and positrons. On this basis they conclude that relativistic jets comprise pair plasmas at least at the radii where they are directly observed.

\subsubsection{Coherent Emission Mechanisms}

All of this calls into question the fundamental synchrotron hypothesis. If the deduced jet powers are unreasonably large then we should certainly be prepared to consider the possibility that the radio emission, or at least its compact and variable part, may be due to a coherent emission mechanism. This is not unreasonable. After all, the sun and Jupiter support high brightness coherent emission under far more docile conditions. Furthermore, a shock front is a very natural environment in which strong and unstable currents are likely to be induced and these are commonly observed to radiate coherently in plasmas. Probably the most likely possibility is coherent cyclotron emission emerging from very much more compact regions than under the jet hypothesis. This requires the magnetic field strength to be hundreds or even thousands of Gauss and so the pressures must be much larger than in the jets. Coherent cyclotron emission is likely to be strongly circularly polarized, unless there are equal numbers of electrons and positrons.

\subsubsection{Microlensing and Refractive Scintillation}

Both microlensing by compact objects in the line-of-sight and refractive scintillation by density fluctuations in the Galactic ionized medium can introduce non-intrinsic variability of the compact structures in jets of extra-galactic radio sources (e.g. knots or shock fronts). For significant variability to occur, these structures must have an angular size of the order the Fresnel scale for scintillation or Einstein radius in the case of microlensing (e.g. Koopmans \& de Bruyn 2000). Both scales are typically several micro-arcseconds. The expected time-scale of variability is determined by the transverse velocity of the source compared to the scattering medium (i.e. the compact objects or the Galactic ionized ISM) and is typically hours to weeks in the case of refractive scintillation or weeks to months in the case of microlensing.

Whereas polarization is typically little affected by either scintillation or microlensing (which retains polarization angle), in both cases only the most compact source structures vary significantly. If these structures have different polarization degrees or angles compared with the flux-density weighted average over the source, the net results will be polarization variability that strongly correlates with the non-intrinsic source variations. A correlation between changes in polarization and non-intrinsic flux variations of extra-galactic radio sources (for example in intra-day variables (IDVs), which strongly scintillate) could therefore provide information on the polarization properties of the most compact micro-arcsecond scale jet structures, which are impossible to observe directly in any other way.

There are several other ways that the Galactic ionized ISM can introduce non-intrinsic variations in the polarization of radio sources (including radio jets), or even induce cir- 
cular polarization. The simplest case is that of extreme scattering events (ESEs), where large localized overdensities in the Galactic ionized medium move into the line-of-sight to the radio source. The enhanced electron column density increases the Faraday rotation and could result in an observable change in the polarization angle of the radio source, as well as in a change of its flux-density due to refractive lensing. Besides Faraday conversion, which converts linear polarization to circular polarization, if a strong gradient in the rotation measure over the source exist, the scintillation patterns of left and right-hand polarized wavefronts will be slightly displaced. This will introduce a time-variable circular polarization that is strongly correlated with the scintillation-induced flux-density variations (Macquart \& Melrose 2000), but independent of the degree of linear polarization (which is not the case for Faraday conversion).

\subsubsection{Inverse Compton Scattering}

A major concern in interpreting the inverse Compton X-ray and $\gamma$-ray observations of blazars is the source of the incident photons. In the lower power objects that can be observed at $\mathrm{TeV}$ energies, these are thought to be synchrotron photons emitted locally within the jet. In the higher power quasars where there is a powerful photoionizing continuum as well as a relativistic jet coming towards us, most of the incident photons are thought to originate from the disk and to be scattered into the jet. However, this is not certain and X-ray (or $\gamma$-ray) polarimetry could validate this, because these scattered $\mathrm{X}$-rays of external origin would be highly polarized.

\subsection{Summary}

- Jets appear to be a common and perhaps even a necessary feature of accreting or possibly even just simply rotating systems.

- Despite much observational progress, the fundamental questions concerning the origin, composition and collimation of jets remain unanswered.

- Black hole jets are formed ultra-relativistically as required to account for their superluminal motion, high radio brightness temperature and prodigious $\gamma$-ray emission.

- Radio polarimetry of relativistic jets is helping us to deduce their composition although coherent emission mechanisms cannot be ruled out.

- X-ray and $\gamma$-ray polarimetry could help refine models of relativistic jets by probing jets close to their origins.

\section{Outflows}

\subsection{Motivation}

Somewhat paradoxically, accreting systems commonly exhibit outflows. The reason for this behavior is simple. As gas accretes onto a compact object it must release its gravitational binding energy. When this is possible, it will do so by radiating. However, this may not be possible when the gas accretes much faster than the Eddington rate, $\dot{M}_{\text {Edd }}=4 \pi G M / \kappa_{T} c$, the photons will be trapped by electron scattering and the energy can only be carried off by a bulk outflow. Even if the radiation is not trapped, then it can still drive an outflow if it encounters gas with an opacity $\kappa>>\kappa_{T}$, for example with dust grains or resonance lines. Either, or more likely both, of these processes are believed to drive the outflows associated with Broad Absorption Line Quasars (BALQs) and this lecture will be primarily about these objects, although the principles involved are more generally applicable. The broad absorption lines by which these quasars are distinguished are associated with the ultraviolet resonance lines of the common ions. (Recall that quasars are mostly at high redshift and so these lines are observed in the 


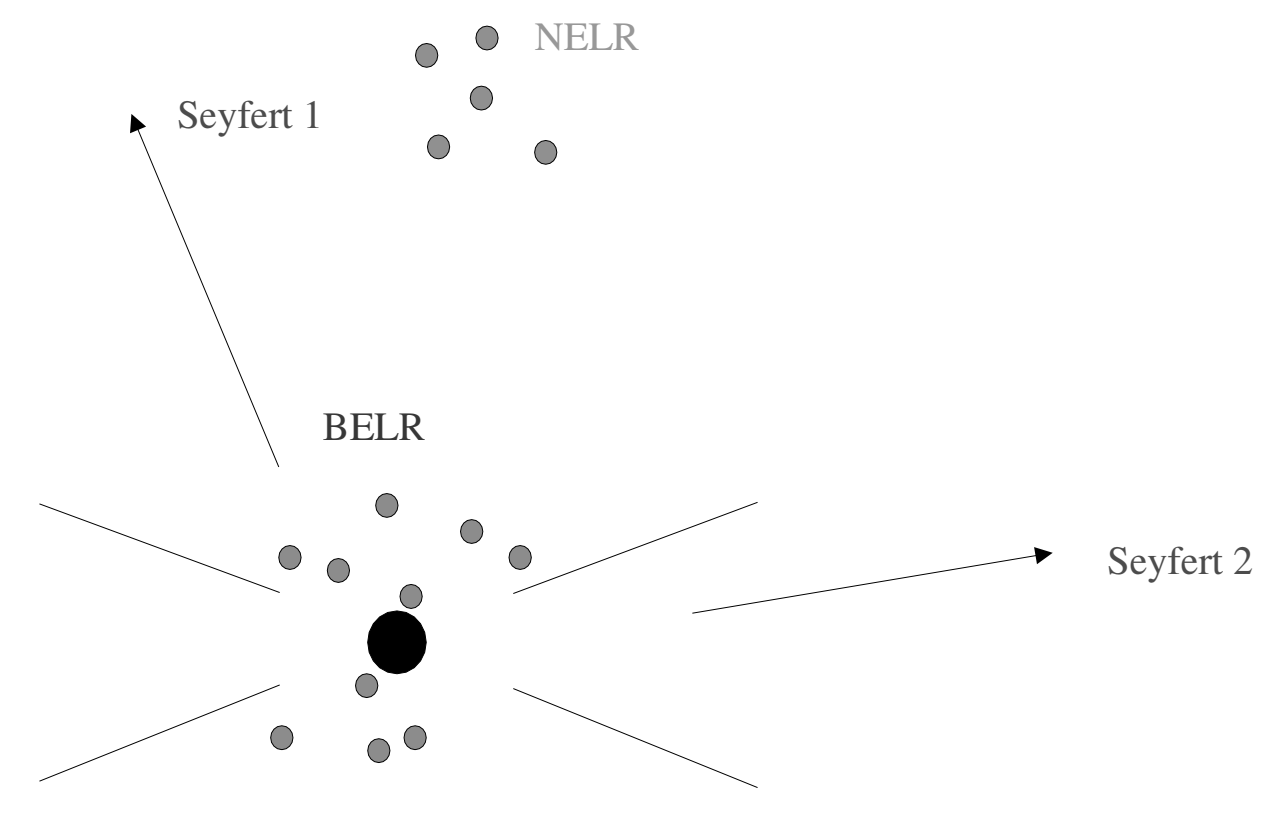

Figure 8. Orientation model for Type 1 and Type 2 Sefert galaxies. In the simplest scheme, only the narrow line region can be seen from a Setfert 2 galaxy; the broad line region lies behind a thick equatorial ring of obscuring gas and dust.

visible.) They show absorption troughs, extending to the blue of the regular, broad emission lines by which quasars are identified spectroscopically. This is just what is also seen in the star P Cygni, though the BALQ relative velocities, $\sim 0.1 c$, which are what one might escape for gas escaping from the vicinity of a black hole, are much larger than those encountered in P Cygni (Weymann et al.1991).

However, we do not understand the flow of gas around the black hole and the location of the emission line and the absorption line gas is still quite controversial. Models of broad line clouds have been constructed in which the gas flows "in, out, round or about" and there may be elements of each of these four kinematic classes in real sources. It is probably safe to conclude that the flow is not very simple; otherwise we would have already understood it using a technique called "reverberation mapping". In this technique it is supposed that the lines vary in direct response to the photoionizing continuum. By monitoring them both, it is possible to construct a Greens function response of the emission line gas, This can then be compared with the predictions of simple kinematic models. It has been possible to use this technique to locate the gas in several instances. However, the details of the velocity field remain controversial.

Despite this, most authors have assumed that the outflow of the absorbing gas is roughly equatorial and radial, originating from close to the hole. This implies that the quasar will only be classified as a BALQ when the observer direction is also close to the equatorial plane; otherwise she will see a regular radio-quiet quasar (Fig. (8)). The goal is to use polarization observations to see if this is truly the case (Antonucci 1993). 


\subsection{Observation}

As we have remarked, a typical BALQ spectrum shows broad emission lines like CIV $\lambda 1548$, accompanied by broad troughs extending to the short wavelength end of the spectrum with widths up to $\sim 30,000 \mathrm{~km} \mathrm{~s}^{-1}$. The emission lines are generally unpolarized, though both the semi-forbidden line CIII] $\lambda 1909$ and Ly $\alpha$ can be polarized, as we shall discuss. The troughs which are caused by approaching gas, represent photons that are removed from the radiation field and scattered sideways. They also remind us that momentum is taken out of the radiation field so that the gas is accelerated (Arav et al.1995). (Of course this may not be the only accelerating force involved, though it is simplest to assume that it is.)

The absorption troughs themselves are not black which presumably means that gas moving along different directions is scattering radiation into our line of sight. In addition, although the troughs are relatively smooth, they do show velocity structure and a variable degree of polarization that can be as large as $\sim 0.2$ a characteristic feature of resonance scattering. However, resonance scattering is not the only means of producing linear polarization; electron scattering can do the job just as well and some observers have preferred this explanation.

In what follows, we shall confine our attention to resonance scattering for two reasons. Firstly, we observe resonance scattering directly and its contribution to the opacity is three to four orders of magnitude larger than that of electron scattering. Secondly, the physics is much more interesting than that of electron scattering! Furthermore we shall confine our attention to the polarization and the implications that it has for the kinematics, as opposed to the dynamics of the flow.

However, we must mention some additional observational clues as to the nature of BALQs. Firstly, BALQs are both radio- and X-ray-quiet. In some models this implies that there is a highly ionized region that can absorb the X-rays and transmit the ultraviolet radiation. In other interpretations, the X-rays are never emitted in the first place. Secondly the BAL phenomenon is pretty much confined to quasars; the lower power Seyfert galaxies do not exhibit these broad troughs. Thirdly, the continuum (after subtracting the galactic contribution) is fairly uniformly polarized with $p \sim 0.01-0.05$, suggesting electron scattering in a disk corona is responsible. This is presumably located inside the absorption line gas. As mentioned above, it is also possible that this same scattering occurs outside the absorption line gas in which case it would probably be responsible for the polarization of the absorption line troughs. Finally, a large polarization, increasing towards shorter wavelength, has been reported at wavelengths longward of the Ly edge in a few, regular quasars. (It should be emphasized, though, that these HST observations were very difficult to make and the results have been controversial.)

\subsection{Physical Processes}

\subsubsection{Resonance Transitions}

Let us first consider the levels of intermediate $\mathrm{Z}$ ions adopting the Russell-Saunders approximation. We can distinguish the Li-like ions, such as CIV, NV, OVI, the Be-like ions, such as CIII, NIV, OV and the B-like ions such as CII, NIII, OIV. The electronic state is determined by the principal quantum numbers for the valence electrons $(n, \ell)$, the total orbital and spin angular momenta $(L, S)$ for the term and the total angular momentum $J$ for the level which, in turn is divided into $2 J+1$ sublevels. This information is encoded in the quantum mechanical designation of the level. For example, the ground state of OIV is $2 s^{2} 2 p^{2} P_{1 / 2}^{o}$ which means that one of the three valence electrons is unpaired in a $2 p$ level with total orbital angular momentum $L=1$ (i.e. a $\mathrm{P}$ state) and 


\section{DOUBLET J=1/2 to $1 / 2,3 / 2$; eg CIV 1558, Ly $\alpha 1216$}

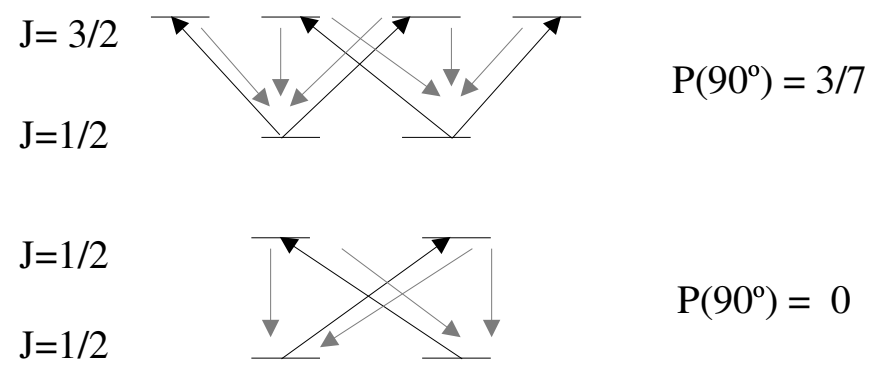

FiguRE 9. Atomic transitions that contribute to the overall polarization for a typical double transition. If the doublet is resolved then the individual components have perpendicular polarizations of $3 / 7$ and 0 . If it is not, then the average polarization is $3 / 11$

total spin $S=1 / 2$, hence the superscript $2 S+1=2$. There are two possible choices for $J$ and the lower energy one, by Hund's rule, has $J=1 / 2$ as designated in the final subscript. (The final superscript, o, indicates an odd parity, which must change under a permitted (electric dipole) transition.) The electric dipole selection rules are that $\Delta \ell= \pm 1 ; \Delta S=0 ; \Delta L=0, \pm 1 ; \Delta J=0, \pm 1$, (except that an $J=0 \rightarrow 0$ transition is forbidden); and $\Delta M=0, \pm 1$, (except that a $M=0 \rightarrow 0$ transition is forbidden).

\subsubsection{Singlets}

Now consider a singlet transition such as the Be-like CIII $\lambda$ 977. The ground state has $J=0$ and only one sublevel with $M=0$; the excited state has $J=1$ and only permitted transitions to $M= \pm 1$ need be considered. The radiation pattern depends only upon the angular parts of that wavefunction, through the Wigner-Eckart theorem. When the scattering angle $\theta=\pi / 2$, the degree of linear polarization can be computed to be $p(\pi / 2)=1$. For a general scattering angle,

$$
p(\theta)=\frac{p(\pi / 2) \sin ^{2} \theta}{1+p(\pi / 2) \cos ^{2} \theta},
$$

just as for Thomson scattering. This formula is generally true for electric dipole transitions and so all we need compute is $p(\pi / 2)$. 


\subsubsection{Doublets}

The next most complicated case is the Li-like doublet transition, eg CIV $\lambda 1550$. Here the ground level has $J=1 / 2$ and there are two possible excited levels of which the lower energy level is $J=1 / 2$. All transitions between sublevels are permitted and the net polarization is $p(\pi / 2)=0$. The higher energy excited level, associated with the shorter wavelength transition, has $J=3 / 2$ and averaging over all of the permitted transitions gives $p(\pi / 2)=3 / 7$. If we average over both transitions according to their statistical weights, then we end up with $p(\pi / 2)=3 / 11$ Fig. (9).

$L y \alpha$, has a similar type of transition. Here the the wavelength separation of the two transitions is so small that the doublet will not be resolved and averaging over the two excited levels makes sense. However, in the case of CIV, the energy difference is larger than the likely thermal width, so that one could, for example, imagine continuum photons propagating out of an expanding flow, encountering the polarizing $J=1 / 2 \rightarrow 3 / 2$ transition first and then encountering the $J=1 / 2 \rightarrow 1 / 2$ transition which erases all of this polarization. It is clear that the polarization is sensitive to the nature of BALQ outflows.

\subsubsection{Triplets}

The next simplest case is the B-like triplet transitions such as CII $\lambda 1335$. In this case, there are two choices for the ground state $J=1 / 2,3 / 2$ and two for the excited state, $J=3 / 2,5 / 2$; the selection rules forbid direct transitions with $J=1 / 2 \rightarrow 5 / 2$. The energy difference between the two ground levels is small enough that they should be equally populated by collisions in the absence of radiative transitions.

It is helpful to introduce the ionization parameter $U$ which is the ratio of the number density of hydrogen ionizing photons to the electron density, designated $n$. When the radiative excitation rate, $\sim 10^{4} U n_{10} \mathrm{~s}^{-1}$, exceeds the collisional excitation rate $\sim 600 n_{10} \mathrm{~s}^{-1}$, the ground state sublevels will be populated in an unequal fashion that must be computed by solving for all the transitions. (Note that both of these rates are likely to be much less than the spontaneous, de-excitation rate and so collisional deexcitation of the excited states is generally thought not to be an issue for permitted lines under AGN conditions.) Under these conditions, when the ground sublevels are radiatively mixed, the resulting polarizations will differ from the values computed assuming as statistical population of the ground sub-levels due to collisions. In this case, the polarization is increased from $p(\pi / 2)=0.21$ to $p(\pi / 2)=0.38$. This increase in predicted polarization is typical.

\subsubsection{Supermultiplets}

The next level of complication arises when an ion in a single ground state can be excited into several different excited states under radiative mixing conditions. In order to solve for the population of the different sublevels and the polarization, we must consider these distinct multiplets together.

\subsubsection{Magnetic Mixing}

A final variation, which is quite likely to be relevant in an AGN, arises when the magnetic field is strong enough that the cyclotron frequency, $\omega_{G}=1.8 \times 10^{7}(B / 1 \mathrm{G}) \mathrm{rad}$ $\mathrm{s}^{-1}$ exceeds the radiative excitation rate. In this case, the relevant eigenstates are referred to the magnetic field direction rather than the normal to the scattering plane and density matrices have to be used to attack the problem in general. This is also known as the Hanle effect and is discussed at greater length here in the solar context by Dr. Stenflo. 
An important consideration is the degree of Faraday polarization. The rotation of the plane of polarization is given by

$$
\Delta \Phi \sim 4 \times 10^{-5}\left(\frac{N}{10^{20} \mathrm{~cm}^{-2}}\right)\left(\frac{B}{1 \mathrm{G}}\right)\left(\frac{\lambda}{1000 \AA}\right)^{2} .
$$

This is unlikely to be a factor at ultraviolet wavelengths, but could be significant in the optical.

\subsection{Interpretation}

\subsubsection{Emission Line Clouds}

There is a standard model of the emission line gas based upon the notion of an emission line cloud, a stratified slab of gas of size $\sim 10^{13} \mathrm{~cm}$, located at a radius $R \sim 0.3 \mathrm{pc}$ from the continuum source with a density $\sim 10^{10} \mathrm{~cm}^{-3}$. The ionization state of the gas is determined by the relative importance of photoionization and recombination which is, in turn, controlled by the ionization parameter, $U$. Typically this is $U \sim 0.1$. These clouds have a photoionization temperature $T \sim 10,000 \mathrm{~K}$ and an equivalent sound speed $\sim 10 \mathrm{~km} \mathrm{~s}^{-1}$. However they are moving with speed $\sim 10,000 \mathrm{~km} \mathrm{~s}^{-1}$ and Mach number $M=1000$ through a hotter and more tenuous confining medium. This is patently absurd! Nonetheless, this model does provide a good representation of the ratios of the observed line strengths. What is clearly required is a convincing dynamical model that retains the successful features of the atomic astrophysics. For the moment, we just consider the polarization in the context of the cloud model.

If we consider a CIV $\lambda 1550$ photon propagating out of a "standard" cloud, the optical depth for solar abundance of carbon, mostly in a triply ionized state, is $\sim 10^{5}$. Under these conditions, photons do not diffuse spatially out of the cloud as might, at first, be guessed. Instead, they undergo a random walk in frequency and escape when they migrate into the wings of the line where the cloud becomes transparent. The polarization really has to be computed using a Monte Carlo simulation and, under these conditions of high optical depth, it is not surprising that it is too small to be measured, even when there is velocity shear giving an anisotropic escape probability. The one conspicuous exception is the semi-forbidden (intercombination line violating the selection rule $\Delta S=0$ ) CIII] line $\lambda 1909$. Here the optical depth is closer to 10 and substantial linear polarization $p \sim 0.01$, was predicted and indeed has been reported in this line (Lee 1994, Cohen et al.1995, but see Ogle et al.1999).

There is a rather different story when external photons are scattered by the emission line clouds. Here we expect a high albedo and roughly half the photons will undergo just one scattering (Korista \& Ferland 1998). If the distribution of scatterers is anisotropic, then we might also expect to detect a linear polarization signal in permitted lines. This is not usually seen, which suggests that a particular line of sight contains at most one cloud at a given wavelength. This is a strong constraint upon models of the velocity distribution.

\subsubsection{Rayleigh Scattering by Lyo}

Hydrogen is the most abundant element so scattering of Ly $\alpha$ photons is likely to occur far into the wings of the line. The fine structure level splitting between $2 P_{3 / 2}$ and $2 P_{1 / 2}$ is quite small for this transition and off-resonance scattering is characterized by the (classical) Rayleigh scattering phase function (e.g. Stenflo 1980). This process is called Rayleigh scattering when the initial and final states of the atom or ion are identical.(This distinguishes it from Raman scattering which arises when they are different.) Rayleigh scattering has been clearly observed in symbiotic stars (Nussbaumer, Schmid, \& Vogel 
1989). For large velocity shifts $\Delta V$, the scattering optical depth exceeds unity for

$$
\frac{\Delta V}{10^{4} \mathrm{~km} \mathrm{~s}^{-1}} \simeq\left[\frac{N_{H I}}{3 \times 10^{22} \mathrm{~cm}^{-2}}\right]^{1 / 2}
$$

(Lee \& Blandford 2000).

Of particular interest is the case of $\operatorname{Ly} \alpha$, because in the damping wings the scattering phase function becomes that of the classical Rayleigh function, which enhances polarization. The fundamental reason why this is the case is that near the line center, where the optical depth is large, most photons migrate in frequency space faster than they do in real space. They are therefore comparatively insensitive to the cloud shape and large scale velocity shear. However, in the wings of the line, the optical depth is much smaller and a large scale pattern in the cloud shapes, for example, translates into a measurable linear polarization. (Effects like this have been reported in high column density supershells associated with starburst galaxies (Lee \& Ahn 1998).)

If the accretion disk of a quasar is warped so that some part of it is shadowed from the direct exposure to the central engine or has a thickness that increases slower than linearly with radius, a large column density $N_{H I}$ may exist in the shaded region and Rayleigh reflection of Ly $\alpha$ is expected. In this case we may expect up to 10 percent polarization in the Ly $\alpha$ wings, which is consistent with $\sim 7$ percent polarization reported from the radio-quiet quasar PG 1630+377 (Koratkar et al.1995)

\subsubsection{Absorption Line Clouds}

A somewhat analogous situation is found for the absorbing clouds observed directly in the BALQs. These are believed to be located outside the emission line region where the ionization parameter $U \sim 1$ and the size is estimated to be even smaller than the size of the emission line clouds $\sim(s / V)^{2} R \sim 10^{11} \mathrm{~cm}$. (In a quite different type of model, it has been proposed that the emitting and absorbing gas originates from very much smaller radii and forms part of a space-filling flow, Murray et al.1995. Many of the following considerations apply to this model as well.)

The actual kinematics of line formation can be quite complicated. This is generally handled under the Sobolev approximation (Rybicki \& Hummer 1978). An incident continuum photon is scattered when it is resonant with a permitted transition taking place in the rest frame of the outflowing gas. The scattered line is redshifted in frequency by $V_{\|} / \lambda$. The surface occupied by gas resonant with a fixed observer frequency is called a Sobolev surface and can have a fairly convoluted shape. Some photons may encounter several Sobolev surfaces before escaping for good. The optical depth to absorption by ion $X$ through a single Sobolev surface where the parallel velocity varies monotonically is

$$
\tau=\int d s n_{X} \sigma=\left|\frac{d V_{\|}}{d s}\right|^{-1} \int d V_{\|} n_{X} \sigma=\left|\frac{d V_{\|}}{d s}\right|^{-1} \frac{c}{\nu} n_{X} \int d \nu \sigma(\nu) .
$$

Substituting numerical values,

$$
\tau=0.3 X_{-4} f \frac{\lambda}{1000 \AA} \frac{d N_{20}}{d V_{9}},
$$

where $X=10^{-4} X_{-4}$ is the abundance of the ion, $f$ is the oscillator strength and $d N_{20} / d V_{9}$ is the hydrogen column density (in units of $10^{20} \mathrm{~cm}^{-2}$ ) per unit velocity (in units of $10,000 \mathrm{~km} \mathrm{~s}^{-1}$ ). Resonance line scattering by the common ions occurs at column densities about three orders of magnitude smaller than those required for electron scattering (Lee \& Blandford 1997).

We can compute the polarization expected from a particular model using Monte Carlo 
simulations. It turns out to be possible to give factor 2 estimates for the polarization, by multiplying expressions which describe the most important factors in producing the integrated polarization,

$$
p \sim p(\pi / 2) D(\tau) A G
$$

In this equation, $D(\tau)$ is a depolarizing factor that takes into account multiple scattering. It is typically roughly fit by an exponential $D=\exp (-\tau / b) . A$ is a factor that takes into account the anisotropy in the escape probability. If the flow is uniformly expanding, it will be very difficult for the photons to escape in the radial direction and far easier for them to escape tangentially. This roughly doubles the polarization. Conversely, if the outflow is in the form of a jet, then small scattering angles will be favored with lower net polarization. Finally $G$ is a geometrical factor which is supposed to account for the observed gas distribution. If the outflow is confined to an equatorial fan subtending a solid angle $\Delta \Omega$, then $G \sim \Delta \Omega / 3$ and the electric vector will lie parallel to the projected symmetry axis. For a jet, the polarization will be perpendicular to the axis (which should be coincident with the radio axis). More extensive observations than have been possible so far will be needed to test the hypothesis that the lines are due to resonance scattering, through the dependence on atomic type (through $p(\pi / 2)$ ), and to decide upon the flow geometry. On this basis, the strongly polarized lines are expected to be HeI $\lambda 584$, OV $\lambda 630$, Ne I $\lambda 736$, NIV $\lambda 765$, CIII $\lambda 977$, SiIII $\lambda 1206$, AlIII $\lambda 1671$ and $\operatorname{MgI} \lambda 2852$. The modestly polarized lines include CII $\lambda 687$, OIV $\lambda 789$ and NIII $\lambda 991$ and weakly polarized lines include OVI $\lambda 1034$, HI $\lambda 1216$, NV $\lambda 1240$, SiIV $\lambda 1396, \operatorname{CIV} \lambda 1549$ and $\operatorname{MgIII} \lambda 2798$. CII $\lambda 858$, NIII $\lambda 764$, and OIV $\lambda 609$ are expected to be unpolarized and can be used to measure the amount of electron scattering.

\subsubsection{Polarization beyond the Lyman Edge}

Observations of the rest ultraviolet continuum from a few high redshift quasars have shown a strong polarization increasing irregularly to shorter wavelengths shortward of the Lyman continuum (Koratkar et al.1998). This may be as large as $p \sim 0.2$, although the observations were extremely difficult and are consequently a bit uncertain. One possible explanation is that there are several highly polarizing singlet lines in this region, like HeI $\lambda 584$, OV $\lambda 630$, NeI $\lambda 736$, NIV $\lambda 765$, or CIII $\lambda 977$. If the outflow speeds associated with these relatively high ionization lines are large, $V \sim 0.1 c$, then it is possible that the lines could overlap enough to give an apparent continuum polarization. It would be good to have the capability to repeat these observations.

\subsection{Summary}

- Spectropolarimetry provides a powerful diagnostic of the disposition of the broad emission and absorption line gas in quasars.

- Resonance scattering should be variably polarized with the degree and direction dictated by fundamental considerations of atomic astrophysics and the flow geometry. The radiation observed in the troughs may be the scattered photons removed from other lines of sight.

- By contrast, the optical continuum exhibits a fairly constant polarization, suggestive of electron scattering.

- Emission lines are generally unpolarized, excepting the semi-forbidden line CIII] $\lambda 1909$. This is consistent with the standard cloud model of emission line formation. BAL troughs are variably polarized.

- Large polarization rising with frequency has been reported to the blue of the Lyman continuum. This may be due to blends of strongly polarized, prominent singlet transitions 


\section{Neutron Stars}

\subsection{Motivation}

Although physicists and astronomers (most famously Baade and Zwicky) were quick to appreciate the possibility that $\sim 10^{57}$ neutrons could assemble to form a self-gravitating neutron star, it was not until the discovery of radio pulsars in 1967, that there was compelling evidence that they really existed. To date we have cataloged over a thousand radio pulsars, know of hundreds of accretion-powered neutron stars in X-ray binaries, and are starting to find isolated neutron stars accreting from the interstellar medium. In addition, five radio, or rotation-powered, pulsars are observed to pulse at optical wavelengths (Chakrabarty \& Kaspi 1998), at least seven as $\gamma$-ray pulsars (Thompson 2000 ), and $\sim 40$ are detectable at X-ray energies (Becker, 2000).

For the astronomer neutron stars are the most common result of evolution of a massive star. However, far from being an endpoint, they represent a rebirth often in a more luminous state than the progenitor star. For the physicist, neutron stars provide a magnificent cosmic laboratory, allowing us to witness the behavior of cold nuclear matter at supranuclear densities, the indirect effects of extremely high $T_{C}$ conductivity and superfluidity, and, as we shall see, the consequences of magnetic field strengths perhaps nine orders of magnitude greater than we can sustain on earth.

Neutron stars are also of special interest to the polarimetrist as they have already furnished the strongest and most rewardingly variable signals of any cosmic sources. It is possible to follow the change in the polarization (sometimes nearly completely polarized) through individual pulses from bright radio pulsars. It is also possible to study the average polarization properties of large samples of pulsars, viewed from a range of vantage points and, thereby build up a picture of the magnetic field geometry and try to determine the site of and the mechanism for their high brightness emission. Unfortunately, there is still no polarimetric capability at X-ray wavelengths where accreting neutron stars in binary systems emit most of their radiation. However, very strong linear polarization is anticipated and the details should be no less prescriptive of the emission.

A third class of object, in addition to the accretion- and rotation-powered pulsars that is of particular interest at the moment is the magnetar. It appears that a minority of neutron stars are formed with super-strong magnetic field $\sim 10^{14}-10^{15}$ G. As predicted by Thomson and Duncan (1995), these magnetars decelerate very quickly but still have a larger reservoir of magnetic energy that can be tapped to power $\gamma$-ray bursts. These field strengths are well in excess of the quantum electrodynamical critical field $B_{c} \equiv m_{e}^{2} c^{3} / e \hbar=4.4 \times 10^{13} \mathrm{G}$, where the cyclotron energy of an electron equals its rest mass. This, in principle, allows us to test the theory in a regime that is qualitatively quite different from that in which impressively high precision tests have already been made. (There is no real anxiety that the theory is suspect above the critical field but, as is the case with general relativity, there is a strong interest in performing the check.) Another class of X-ray source is the Anomalous X-ray Pulsars. These are possibly a late evolutionary phase of magnetars.

In this section, we shall discuss the expected polarimetric properties of all three types of sources, although there are only observations of rotation-powered pulsars.

\subsection{Observation}

\subsubsection{Rotation-Powered Pulsars}

Radio pulsars are spinning, magnetised neutron stars (e.g. Lyne \& Smith 1998). The majority have spin period between $0.1 \mathrm{~s}$ and $3 \mathrm{~s}$ and surface magnetic field strengths $\sim 10^{12} \mathrm{G}$, estimated from the rate at which they appear to slow down. The neutron stars 
themselves appear to be mostly formed with masses quite close to the Chandrasekhar mass $\sim 1.4 \mathrm{M}_{\odot}$. Their poorly measured radii are $\sim 10 \mathrm{~km}$, consistent with there having central densities a few times nuclear as the best models of the nuclear equation of state imply. (We really do not know the interior composition at all well. It could be mostly neutrons or contain a large fraction of protons, hyperons, pions or even free quarks. Accurate measurements of the radius along with the rate of cooling will provide important constraints on the equation of state of nuclear matter.) Other, impressive vital statistics of neutron stars include escape velocities $\sim 0.3 c$, surface gravities $\sim 10^{14} \mathrm{~cm} \mathrm{~s}^{-2}$ and maximum spin frequencies (that are nearly attained in observed objects) $\sim 1 \mathrm{kHz}$. The radio emission from pulsars has extremely high brightness temperatures which can, by some estimates, exceed $\sim 10^{30} \mathrm{~K}$.

The integrated pulse profiles of radio pulsars frequently show one, two or three pulses. This, and other observational evidence, has been interpreted in terms of an emission model where there is a strong "core" beam of emission close to the magnetic axis surrounded by a weaker "cone" beam. When the observer latitude is similar to that of the magnetic axis, a single, dominant core component is seen. Increasing (or decreasing) the observer latitude leads to a three peaked, cone-core-cone pattern. When the observer is more inclined to the magnetic axis, only the two cone components will be seen. These pulse profiles exhibit strong, broad band linear polarization varying through the pulse. Values $p \sim 1$ are consistently measured at certain pulse phases in certain pulsars. The position angle swings regularly through the main pulse with a total swing that can be as high as $\sim 180^{\circ}$. Strong circular polarization is also commonly measured near the center of the pulse, with the handedness often changing sign.

Individual pulses, whose polarimetric properties can be measured in the strongest pulsars, are no less interesting. They show individual emission units, known as subpulses, with durations typically a few degrees of pulsational phase. These can "drift" through the pulses appearing at progressively earlier or later phases in successive pulses. These subpulses often exist in one of two orthogonal polarization states. Even shorter timescale features known as microstructure (or now even nanostructure) has been well documented and these too can exhibit high, though complex, polarization properties.

The optical pulses from the Crab pulsar in the Crab Nebula have been particularly well studied. The pulse profile is cusp-like and the plane of polarization swings smoothly through $\sim 70^{\circ}$, while the degree varies between $p=0.1$ and 0.5 . Another famous optical pulsar is associated with the Vela supernova remnant, shows similar strong, variable linear polarization.

\subsection{Physical Processes}

\subsubsection{Curvature Radiation}

The large measured brightness temperatures imply that the radio emission is produced by a coherent process. One widely discussed possibility is that the emission is some variant on coherent curvature emission whereby bunches of charged particles stream outward along the curving, roughly dipolar magnetic field lines from the star with ultrarelativistic speed (Lorentz factors $\gamma$ of several hundred) and radiate like giant electrons. The emission properties are like those already summarized for synchrotron radiation, with the important difference that the radius of curvature of the orbit, $R$, is energy-independent. The characteristic emission frequency is $\omega \sim \gamma^{3} c / R$, lying in the radio band for $R \sim 10-100 \mathrm{~km}$. The radiation from an individual bunch is beamed within an angle $\sim \gamma^{-1}$ to the direction of motion.

At a particular pulse phase, the observer sees emission from a curve though the magnetosphere where the line of sight is tangent to the magnetic field. There is thought to be 
a radius-to-frequency mapping so that the emission at a given frequency is concentrated over an interval of radius along this curve, and that this radius decreases with increasing frequency. The polarization from a tangent point will be quite strongly linearly polarized with electric vector parallel to the projected curvature vector on the sky. As the pulsar spins, this projected curvature vector will rotate on the sky and a characteristic swing of the plane of polarization will be produced. This is known as the rotating vector model. If we view an individual bunch from one side of its orbital plane then the other, we will see one sense of circular polarization followed by the opposite sense. This mechanism clearly has the ingredients to explain the radio polarization observations. However, it is a bit puzzling how a totally polarized pulse can be formed in this manner. One possible explanation is that the bunches are quite strongly flattened and they radiate most strongly perpendicular to their flattening plane, where the polarization will be most strongly linear.

\subsubsection{Maser Processes}

There has recently been a resurgence of interest in maser emission models. It is relatively easy to imagine that the necessary population inversion will develop in the outflowing plasma. A typical pulsar can develop an EMF of $\sim 10^{13}-10^{16} \mathrm{~V}$ and a small fraction of this potential difference developing in a transient "gap" will create a fast stream of electrons and/or positrons that can stream through more slowly moving (though still ultrarelativistic) plasma. What is a bit harder is to find suitable wave-particle interactions that can lead to an overall negative absorption coefficient. As an illustration of the difficulty, note that synchrotron radiation in vacuo has an absorption coefficient

$$
\kappa=\frac{1}{8 \pi \nu^{2}} \int d N \frac{1}{\gamma^{2}} \frac{\partial}{\partial \gamma} \gamma^{2} p_{\nu}(\nu, \gamma) .
$$

The single particle emissivity, $p_{\nu}(\nu, \gamma)$ is proportional to $\nu^{1 / 3} \gamma^{-2 / 3}$ at low frequency and increases with energy at high frequency. Hence, synchrotron absorption is necessarily positive and maser action is precluded, independent of the particle distribution function. Similar conclusions have been drawn for other emission mechanisms in vacuum.

However this conclusion does not necessarily follow if there is a plasma present. One particularly interesting case is the so-called anomalous cyclotron (otherwise known as cyclotron-Cerenkov) resonance between a wave with angular frequency $\omega$ and wave vector $\vec{k}$ interacting with an electron (or positron) moving with velocity $\vec{v}$, in a field where the non-relativistic gyro frequency is $\omega_{G}$. This occurs if

$$
\omega-k_{\|} v_{\|}=-\omega_{G} / \gamma \text {. }
$$

where $\|$ refers to the component along the magnetostatic field.

This equation needs some interpretation (Lyutikov, Blandford \& Machabeli 1999). Consider, for simplicity, a circular polarized wave propagating along the magnetic field. A resonance satisfying the condition Eq. (4.40) clearly requires that the phase velocity of the wave be less than $c$. If we transform into the guiding-center frame of the electron, then the wave angular frequency changes sign indicating that it is propagating in the opposite direction along the magnetic field, with the same sense of circular polarization. This means that it resonates with particles gyrating in the opposite sense around the field (i.e. with opposite charge) than is the case in a regular cyclotron resonance. Put another way the particle outruns the wave so that, in the frame where the wave is at rest, the particle follows the electric vector as it spirals around the magnetic field. A consequence is that, in the electron guiding-center frame, a quantum of wave energy has negative energy. Therefore in exciting the electron to a higher state of gyration it emits 


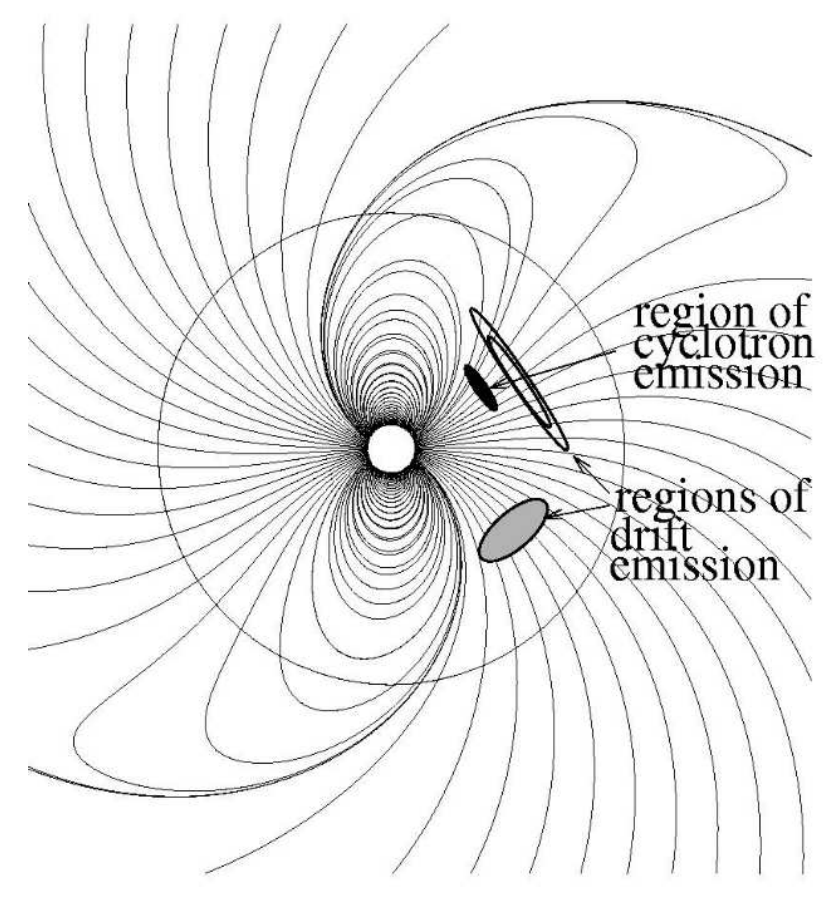

FiguRE 10. Location of core and conal emission regions in a maser model of pulsar emission (after Lyutikov et al.1999).

a quantum and vice versa. (In the rest frame, population inversion now requires having more particles in the lower gyrational state!) Not surprisingly, this arrangement can lead to maser action in the outer magnetosphere and this has been proposed as a mechanism to produce the core emission. The modes are naturally circular polarized, although the handedness depends upon the details of the electron and positron distribution functions.

This is not the only possible way to have a maser process. There is a second resonance associated with the curvature drift motion $v_{\text {drift }}$ of the gyrating electron as it moves along the curving magnetic field

$$
\omega-k_{\|} v_{\|}=k_{\perp} v_{\text {drift }}
$$

This will be perpendicular to the curvature plane and will consequently produce emission with polarization orthogonal to that predicted by the rotating vector model. This mechanism has been invoked to account for the cone emission (Fig. (10)).

\subsubsection{Propagation Effects}

There is unfortunately a complication (Arons \& Barnard 1986, Lyutikov et al.1999, Hirano \& Gwinn 2001). The emitted radiation must propagate through the outer magnetosphere. This can lead to genuine absorption at the normal cyclotron resonance by more slowly moving electrons. Landau damping is also a possibility. Furthermore, there are refractive effects that may imprint additional polarization on the emergent radiation in much the same way as occurs in the ionosphere. There can be mode conversion, for example from a subluminal ordinary mode to a propagating electromagnetic wave. Finally, and perhaps most interestingly from a physics perspective, there can be non-linear 
scattering effects (Lyutikov, 1998). At the high brightness temperature (or, equivalently, large occupancies of individual quantum mechanical states) found in pulsar radiation there will be a large amount of scattering between different radiation beams. These can be mediated by individual electrons, in which case the interaction is known as induced Compton scattering, or by collective wave modes of the plasma (known as Raman scattering when the scatterer is an electrostatic wave). There is not space to discuss these rather complex processes further, save to remark that, the associated matrix elements have a strong sensitivity to polarization and frequency and that this can be used to identify them. The theory is starting to match the observations in its richness!

Some of these propagation effects can also be relevant in the interstellar medium.

\subsubsection{Thomson Scattering in a Strong Magnetic Field}

The cross section for Thomson scattering must be changed if there is a strong magnetic field present (e.g. Mészáros 1992). In the limit when the wave angular frequency $\omega<<$ $\omega_{G}$, or $E<<44\left(B / 10^{12} \mathrm{G}\right) \mathrm{keV}$, the electrons are constrained to move along the magnetic field like beads on a wire. The dominant cross section is between polarization states with $\vec{k}, \vec{E}, \vec{B}$ coplanar. It is clearly given by

$$
\frac{d \sigma}{d \Omega}=r_{e}^{2} \sin ^{2} \theta \sin ^{2} \theta^{\prime},
$$

where $\theta, \theta^{\prime}$ are the angles made by the incident and scattered wave vectors with the magnetostatic field.

\subsubsection{Inverse Compton Scattering in a Strong Magnetic Field}

This anisotropy in the cross section introduces an additional complication to inverse Compton radiation. This is because the incident photon propagates in a direction making an angle $\sim \gamma^{-1}$ to the magnetic field and so the total scattering cross section as given by Eq. 4.42 is reduced by a factor $\sim \gamma^{-2}$ from the Thomson value assuming that the frequency in the electron rest frame $\left(\omega^{\prime}\right)$ is less than $\omega_{G}$. However, under these circumstances, we must also consider the effect of the " $\vec{E} \times \vec{B}$ " drift of the electron perpendicular to the magnetic field. This will produce an oscillatory electron motion that is larger than the field parallel motion by a factor $\gamma \omega^{\prime} / \omega_{G}$. The azimuth and incident polarization-averaged cross section will be given by

$$
\frac{d \sigma}{d \Omega}=\frac{r_{e}^{2}}{4}\left(\frac{\omega}{\omega_{G}}\right)^{2}\left(1+\cos ^{2} \theta\right)\left(1+\cos ^{2} \theta^{\prime}\right),
$$

for incident frequency $\omega$ satisfying $\omega_{G} / \gamma \omega<\omega_{G}$. In practice, this leads to a rather complex polarization pattern.

\subsubsection{Quantum Electrodynamical Effects}

The virtual electron-positron plasma that comprises the QED vacuum affects the propagation of radiation through it. These are clearly likely to be of importance when the magnetic field strength approaches the critical field strength, $B_{c}$.

An external magnetic field causes photons of different polarizations to travel at slightly different speeds. The $C P$-invariance of electrodynamics tells us that the two modes must be linearly polarized. Specifically, the indices of refraction of both modes differ from unity and are given by (Heyl \& Hernquist 1997a):

$$
n_{\perp}=1+\frac{\alpha}{4 \pi} \frac{8}{45}\left(\frac{B_{\perp}}{B_{c}}\right)^{2}+\cdots,
$$




$$
n_{\|}=1+\frac{\alpha}{4 \pi} \frac{14}{45}\left(\frac{B_{\perp}}{B_{c}}\right)^{2}+\cdots,
$$

for $\hbar \omega \ll m_{e} c^{2}$ and $B \ll B_{c}$. $B_{\perp}$ is the component of the magnetic field perpendicular to the propagation direction of the photon. A photon in the perpendicular polarization has its electric field vector perpendicular to the projection of the magnetic field into the transverse plane, and similarly for the parallel polarization.

For fields stronger than $B_{c}$, the index of refraction for the mode with the electric field perpendicular to the external magnetic field saturates at

$$
n_{\perp}=1+\frac{\alpha}{4 \pi} \frac{2}{3} \sin ^{2} \theta+\cdots,
$$

while the index for the other mode increases without limit

$$
n_{\|}=1+\frac{\alpha}{4 \pi} \frac{2}{3} \sin ^{2} \theta \frac{B}{B_{c}}+\cdots .
$$

Therefore, there are two natural limits to the behavior. In the weak field regime the vacuum polarization may be sufficiently strong to decouple the polarization states as they propagate through the magnetosphere (Heyl \& Shaviv 2000, 2001). Normal pulsars as well as strongly magnetized white dwarfs fall in this regime. In the strong-field regime magnification and distortion of the image of the neutron star surface may become important in addition to the decoupling of the propagating modes (Shaviv, Heyl \& Lithwick 1999).

The processes of photon splitting and one-photon pair production are forbidden in fieldfree regions, the first by Furry's theorem and the second by four-momentum conservation. However, in the strong magnetic fields surrounding a neutron star, both processes may be important. Photon splitting most strongly affects photons in the $\perp$-mode which may split into two photons in the $\|$-mode (Adler 1971, the mode-naming convention used here is opposite to that used by Adler); this both distorts and polarizes the photon spectrum.

One-photon pair production has an energy threshold of $\hbar \omega \sin \theta>2 m_{e} c^{2}$ for photons in the $\|$-mode. The threshold for photons in the $\perp$-mode is slightly larger $\hbar \omega \sin \theta>$ $m_{e} c^{2}\left(1+\sqrt{1+2 B / B_{c}}\right)$. Near the thresholds, in strong magnetic field especially, the cross-section for this process is complicated by the formation of the pair in discrete Landau levels or a positronium bound state (Daugherty \& Harding 1983; Usov \& Melrose 1996).

\subsection{Interpretation}

\subsubsection{Rotation-powered Pulsars}

Partly because it provides such a straightforward interpretation of the polarization data, the curvature radiation model is probably the favorite explanation for pulsar radio emission. However, it does have some drawbacks. One of these is that it is difficult to maintain a compact bunch for very long as the electrons travel along trajectories with different radii of curvature and are subject to radiation reaction. Another problem is that it has proven hard to find a suitable plasma instability which will allow charge particle bunches to grow. A third and currently controversial observational claim is that the emitting area is much larger than expected if the bunches form in the inner magnetosphere as the rotating vector model requires. Specifically, Gwinn, et al.(2000) find that the size of the Vela pulsar emission region is roughly ten per cent of its light cylinder radius, $\sim c / \Omega$, suggesting that the emission originates in the outer magnetosphere. Conversely, Cordes (2001) finds that the source is unresolved.

The maser explanation, by contrast, only works in the outer magnetosphere and the average direction of polarization should be orthogonal to the projection of the pulsar 
spin axis on the sky. This can be tested using the Crab and Vela pulsars where X-ray jets are observed which, although their formation is not understood, are presumed to be along the projected spin axis. In the case of the Crab pulsar, the situation is ambiguous because it is unclear if the two pulses come from one or two magnetic poles. However, in the case of the Vela pulsar, the electric vector is unambiguously perpendicular to the projected spin axis, consistent with the maser model. It could also be consistent with curvature radiation if propagation effects are important. It should be possible to discriminate between these two models observationally.

The pulsed optical radiation and X-ray radiation seen from several radio pulsars is generally thought to be incoherent synchrotron and inverse Compton radiation, respectively. However, the location of the emission region, and, in the case of the X-rays, the source of the incident photons (the surface of the star or coherent radio emission from the magnetosphere) is currently undecided. Suffice it to remark it here that polarization arguments figure prominently in these debates.

\subsubsection{Accretion-powered Pulsars}

In addition to being strongly polarizing, the opacity, Eq. 4.42, is highly anisotropic. So, even though we cannot measure the X-ray polarization directly, at present, it does have a strong effect on what we observe. In particular, the mass that accretes onto a spinning neutron star with a surface field $\sim 10^{12} \mathrm{G}$ is likely to be channeled toward the magnetic poles and, if the accretion rate is large enough, there will be a significant Thomson opacity at the poles. However, it will be much easier for the radiation to escape along the direction of the magnetic field than in the transverse direction. For this reason, $\mathrm{X}$-rays are thought to emerge in two broad pencil beams about the magnetic axis as is observed.

\subsubsection{Surface Emission from Isolated Neutron Stars and Magnetars}

The atmospheres of neutron stars are thought to emit strongly polarized radiation (Pavlov \& Shibanov 1978). The opacities in the two polarization modes of the atmospheric plasma may differ by several orders of magnitude (Lodenqual et al.1974). The opacity in the extraordinary mode (i.e. $E \perp B)$ is generally a factor of $\left(\omega / \omega_{G}\right)^{2}$ smaller

than in the ordinary mode. Since the atmospheres are typically at a temperature of several million degrees, the natural place to study this emission is in the X-rays. Furthermore, as we shall see, the vacuum significantly affects the propagation of radiation passing through it at X-ray and higher energies.

Although the emission at the surface may nearly be fully polarized, one observes radiation from regions with various magnetic field directions. In this vein, Pavlov \& Zavlin (2000) argue that the net polarization in the X-rays is on the order of ten percent and decreases for more compact stars. However, this treatment ignores the fact that QED renders the vacuum birefringent. The field strength varies sufficiently gradually, that is

$$
\frac{\nabla|\Delta k|}{|\Delta k|} \ll|\Delta k|,
$$

where

$$
|\Delta k|=\frac{\alpha}{4 \pi} \frac{2}{15} \frac{\omega}{c}\left(\frac{B_{\perp}}{B_{k}}\right)^{2},
$$

in the weak field regime, that the two polarization modes are decoupled. Radiation produced at the surface with its polarization direction perpendicular to the local magnetic field direction will keep its polarization perpendicular to the field even as it passed through regions where the field direction changes. The observed polarization reflects 
the direction of the field at a distance

$$
r \approx 1.2 \times 10^{7}\left(\frac{\mu}{10^{30} \mathrm{Gcm}^{3}}\right)^{2 / 5}\left(\frac{\nu}{10^{17} \mathrm{~Hz}}\right)^{1 / 5}(\sin \beta)^{2 / 5} \mathrm{~cm},
$$

from the center of the star (Heyl \& Shaviv 2000, 2001). Here $\mu$ is the magnetic dipole moment of the neutron star, and $\beta$ is the angle between the dipole axis and the line of sight. QED ensures that the strongly polarized radiation at the surface of the neutron star remains strongly polarized until it is detected; therefore, the simple detection of strongly polarized X-rays from the atmosphere of a neutron star will verify a thus far untested prediction of QED. Cheng \& Ruderman (1979) used a similar argument to account for the strong polarization of radio emission from pulsars.

Although, the QED process of one-photon pair production plays a crucial role in radio pulsars by fueling the plasma that produces the emission (e.g. Daugherty \& Harding, 1982), the threshold for the reaction is much higher than the typical energies from the surface emission. The cross-section for photon splitting increases dramatically with increasing photon energy, $\propto E^{6}$ (Heyl \& Hernquist 1997b) and is only important above $10 \mathrm{keV}$ even in the strongest magnetized sources.

\subsection{Soft-Gamma Repeaters}

Thompson and Duncan first argued that the soft-gamma repeaters are neutron stars fueled by a dynamic magnetic field whose strength greatly exceeds $B_{c}$. In their quiescent state, these objects emit thermal radiation from their surfaces and the discussion of the previous subsection applies. If their surface fields are sufficiently strong (the surface field is expected to exceed the value estimated by spin down of $\sim 10^{15} \mathrm{G}$ ), magnetic lensing may be important for photons whose polarization is parallel to the magnetic field (i.e. the ordinary mode); however, thermal emission in this mode appears to be strongly suppressed.

However, what makes the soft-gamma repeaters unique is that they burst. In fact, the soft-gamma repeater, SGR $1900+14$, is the only object beyond our solar system to have had contemporary geophysical consequences (it ionized the nightside upper atmosphere nearly to daytime levels). This soft gamma-ray emission is generally well below the threshold for one-photon pair production, but photon splitting should degrade the energies of the photons by at least a factor of two and polarize them by converting photons in the extraordinary mode to the ordinary mode (Baring \& Harding 1997). Observing this tracer of photon splitting would require gamma-ray polarimetry.

\subsection{Summary}

- Magnetized neutron stars provide cosmic laboratories where we can observe unique polarization effects in action and use them to identify the emission mechanism.

- Radio pulsars offer the richest polarization data set outside the solar system. They are strongly diagnostic of the emission mechanism and the effects of propagation.

- Accretion-powered pulsars introduce new effects associated with strong field anisotropic emission and scattering in the $\sim 10^{12} \mathrm{G}$ surface fields. Even though the X-ray polarization is not yet measured, it is important in determining the total spectrum and pulse profile.

- X-ray pulsars allow us to address important physics questions, like the composition and compressibility of cold matter at supra-nuclear density.

- The simple detection of strongly polarized X-rays from the atmosphere of a neutron star (or optical radiation from neutron stars with $B>10^{13} \mathrm{G}$ ) will verify the prediction 
that QED renders the vacuum birefringent and provide an estimate of the radius of the star itself.

- The convincing case that magnetars exist with surface fields well in excess of the critical field, $\left(4.4 \times 10^{13} \mathrm{G}\right)$, offers the equally exciting, (though observationally very challenging) prospect of testing quantum electrodynamics in a regime far removed from terrestrial investigation.

\section{Black Holes}

\subsection{Motivation}

There is now very good evidence for the existence of black holes in the universe. They appear to be a common endpoint of the evolution of massive stars in our Galaxy and nearby galaxies and we know of roughly ten good cases where the dynamically determined mass significantly exceeds the Oppenheimer-Volkoff limit (for neutron stars) $\sim 2.5 \mathrm{M}_{\odot}$ and the Chandrasekhar limit (for white dwarfs) $\sim 1.4 \mathrm{M}_{\odot}$. In addition, there are many more cases of transient X-ray sources where the circumstantial evidence, in the absence of dynamics, is pretty convincing. A significant fraction of massive stars must end their life this way.

Similarly, dynamical studies of the nuclei of nearby galaxies reveal the presence of "massive dark objects" which, if they were, for example, clusters of compact objects, would be very short-lived. Identifying them with massive black holes is by far the most conservative conclusion to draw. It appears that the nuclei of most normal galaxies, including our own, contain black holes with masses in the range $\sim 3 \times 10^{6}-3 \times 10^{9} \mathrm{M}_{\odot}$. (There are speculative suggestions that there may be a large population of intermediate mass black holes, perhaps relics of the first generation of stars.)

The existence of black holes is, arguably, the most far-reaching implication of the general theory of relativity. The theory has been probed in the weak field regime and passed all quantitative tests with an accuracy that can be as small as $\sim 3 \times 10^{-4}$. It is in the nature of the theory that, if we understand the laws of physics under these circumstances, it is simply a question of geometry to describe strong field environments, when the equivalent Newtonian potential approaches $c^{2}$. If the theory in its essential simplicity is correct, then the metric of an asymptotically flat black hole spacetime (excluding some mathematical niceties) is essentially given. Indeed, in one of the greatest successes of mathematical physics, we have a closed form version of the metric of a spinning black hole, known as the Kerr metric, and essentially all classical physics that can be discussed in a flat spacetime can also be discussed around a black hole; there are no difficulties of principle. There are, however, considerable difficulties in execution (which have mostly been overcome using numerical calculations.) Although we know of more general spacetimes that include a gravitationally significant charge or orbiting mass, we believe that these are irrelevant to observed black holes and that astronomers need only be concerned with the Kerr metric.

However, it is logically possible that the theory of general relativity could be wrong or incomplete and that, as a consequence, black holes are fundamentally and observably different from their general relativistic description. For this reason, it is vitally important that we try to find ways to probe the spacetime around black holes, now that we know where to find them. In this regard, observing black holes provides a far more telling test of relativity theory than cosmological observations. This is because cosmological observations are seriously compromised by our deep ignorance of the nature of dark matter and energy as well as the effects of evolution. 
We already know that gravitational waves exist. Binary pulsars are observed to lose orbital energy at rates that agree with theory to a fraction of a percent. However, this mostly tests linearized theory even in the sources. The direct detection of gravitational waves, which we hope will happen one day, is also is only a linear test. The ultimate test of general relativity is to make detailed observations of gravitational waves from coalescing black holes; an observation that I suspect will be not be technologically feasible for some time. In addition to testing strong field relativity, this can also provide much useful astrophysical information on galaxy merger rates, AGN evolution and so on. Computing the wave forms in necessary generality is a major challenge to computational science. What is relevant in the present context is that gravitational waves have natural polarization states, just like electromagnetic waves, and much of the information from these coalescences will be encoded in the polarization details. (There are other strong field sources of gravitational radiation that have been considered, notably topological defects like cosmic strings. Unlike the case with black holes, there is no observational evidence yet for their existence. However, if they are ever discovered, then it may well be their gravitational radiation polarization that is their distinctive signature. Computing this polarization is a good project which appears to have been mostly ignored.)

There is a second and quite different reason for being interested in black holes. This is that we do not understand properly how they work. We have already introduced jets, disks, and outflows; and discussed how polarization observations can teach us about their properties. We already know, from direct observation, that all three of these continue down to relatively close (in logarithmic terms) to the black hole. This is where most of the energy is released. However, we do not understand how this all happens and how the flow around the black hole depends upon the mass and the spin of the hole as well as the accretion rate and the immediate environment. It appears that the answers to these questions will only be found by exploring the black hole itself.

A third contextual aspect of this study is that we are beginning to suspect that black holes have a much larger and more active role in galactic and extragalactic astronomy than used to be the case. It is increasingly likely that gamma ray bursts are associated with the formation or augmentation of black holes and that these have major environmental impacts on their surroundings and could soon become useful cosmological probes. Black hole transients provide the dominant hard X-ray emission of galaxies like our own and create powerful outflows. The discovery of dormant, or near-dormant black holes in the nuclei of normal galaxies has affirmed the long-standing black hole model of active galactic nuclei, including quasars and giant, double radio sources. However, the implications have much broader implications than the properties of AGN per se. The quasars themselves provide the best cosmologically distant beacons that we have and they allow us to study the intergalactic medium, matter and cosmography. Furthermore, it is becoming increasingly apparent that they have an much more active role in the very formation of galaxies, both in the initiation and perhaps in the cessation of the process.

The long term observational goal, then, is to verify that black holes are described by the Kerr metric and to measure their masses and spins in such a way as to elucidate their role in stellar and galactic evolution. In this section, we will try to show how polarization observations can contribute to meeting this objective.

\subsection{Observation}

We have already described most of the relevant observations of black holes including the fairly strong dynamical measurements of their masses and the Fe K line emission which provides the strongest evidence to date that black holes spin relatively rapidly. The 
most relevant, existing observation for the purpose of this lecture are the measurements of linear and circular polarization.

\subsection{1. $\operatorname{Sgr} A^{*}$}

The center of our Galaxy appears to be identified with the radio source Sgr A*. There is now excellent dynamical evidence that is a "dark, compact object" with a mass $2.6 \times$ $10^{6} \mathrm{M}_{\odot}$ and a black hole is by far the most conservative interpretation. The source is nearly at rest and stars can be tracked moving (and accelerating) around it (Ghez et al.2000). The source has a spectrum which peaks at $\sim 300 \mathrm{GHz}$ and the image is broadened at radio wavelengths by interstellar scattering. At $43 \mathrm{GHz}$, the average scatter-broadened size is reported to be $\sim 3 \times 10^{13} \mathrm{~cm}$, (Lo et al.1999) and to scale roughly $\propto \lambda^{2}$. Sgr $\mathrm{A}^{*}$ is a weak and soft X-ray source. Interestingly, a $106 \mathrm{~d}$ periodicity in the radio emission has also been reported (Zhao, Bower \& Goss 2001).

Sgr $\mathrm{A}^{*}$ has long been known to have negligible linear polarization at radio and $\mathrm{mm}$ wavelengths. This is not a surprise because the Faraday rotation is expected to be quite high so that the differential (in both angle and frequency) rotation is also large enough to depolarize all measurements. What is a surprise (as discussed here by Hildebrand) is that Aitken et al.(2000) measure $\sim 10$ percent linear polarization at $150 \mathrm{GHz}$, using the SCUBA instrument on the James Clerk Maxwell Telescope, although there is an upper limit of $\sim 1$ percent at $86 \mathrm{GHz}$ (Bower et al.2000). Clearly there is a need to confirm the SCUBA measurement.

More recently, it has been discovered that Sgr A* exhibits quite strong circular polarization, (Bower, Falcke \& Backer 2000). The $5 \mathrm{GHz}$ degree of circular polarization appears to have been stable at a value of $\sim-0.003$ for nearly twenty years. At higher frequencies, up to $\sim 43 \mathrm{GHz}$, the degree of circular polarization appears to increase up to a few percent and become increasingly variable, doubling in a few days.

\subsection{Physical Processes}

\subsubsection{Spinning Black Holes}

The spacetime around a spinning black hole is described by the Kerr metric expressed in Boyer-Lindquist coordinates with $G=c=1$

$$
\begin{aligned}
d s^{2}= & -\left(1-2 m r / \rho^{2}\right) d t^{2}-\left(4 a m r \sin ^{2} \theta / \rho^{2}\right) d t d \phi+\left(\rho^{2} / \Delta\right) d r^{2}+\rho^{2} d \theta^{2} \\
& +\left(r^{2}+a^{2}+2 m r a^{2} \sin ^{2} \theta / \rho^{2}\right) \sin ^{2} \theta d \phi^{2}
\end{aligned}
$$

where

$$
\begin{aligned}
\rho^{2} & =r^{2}+a^{2} \cos ^{2} \theta, \\
\Delta & =r^{2}-2 m r+a^{2},
\end{aligned}
$$

and $m$ is the mass such as would be measured by the orbit of a distant satellite, and $a<m$ is the specific angular momentum of the hole, as could be measured operationally by the precession rate of a gyroscope.

There is an event horizon, $\mathcal{H}$, which is located where $\Delta=0$ i.e. where the radial coordinate $r=r_{+}=m+\left(m^{2}-a^{2}\right)^{\frac{1}{2}}$. Particles on timelike or null geodesics must be inwardly moving within $r_{+}$which leads to the interpretation that $\mathcal{H}$ represents a surface of no return. The four velocity, $\vec{u}=\{d t / d \tau, d r / d \tau, d \theta / d \tau, d \phi / d \tau\}$ of a material particle satisfies

$$
g_{\alpha \beta} u^{\alpha} u^{\beta}=-1 \text {. }
$$

The equation of a photon, following a null geodesic is given by $g_{\alpha, \beta} d x^{\alpha} d x^{\beta}=0$, supple- 
mented with equations representing the conservation of energy and angular momentum as well as an additional integral of the motion.

The angular velocity $\Omega=d \phi / d t$ of a particle, orbiting with fixed $r, \theta$, therefore satisfies

$$
u^{02}\left[g_{00}+2 \Omega g_{0 \phi}+\Omega^{2} g_{\phi \phi}\right]=-1 .
$$

This implies that $\Omega_{\min }<\Omega<\Omega_{\max }$ where $\Omega_{\min }>0$ when $r_{+}<r<r_{e} \equiv m+\left(m^{2}-\right.$ $\left.a^{2} \cos ^{2} \theta\right)^{1 / 2}$. The radius $r_{e}$ is known as the static limit and the region between it and the horizon, where inertial frames are dragged by the spin of the hole, is known as the ergosphere. A particular significance of the ergosphere is that orbits of negative energy (including rest mass) exist within it. As $r \rightarrow r_{+}$at the event horizon,

$$
\Omega_{\min } \rightarrow \Omega_{\max } \rightarrow \Omega_{H} \equiv a /\left(r_{+}^{2}+a^{2}\right)^{1 / 2},
$$

the angular velocity of the hole.

A remarkable theorem due to Hawking states that the area of the horizon, which can be computed from the metric to be $A=\int_{\mathcal{H}}\left(g_{\theta \theta} g_{\phi \phi}\right)^{1 / 2} d \theta d \phi=4 \pi\left(r_{+}^{2}+a^{2}\right)$ cannot decrease. We can use this to define a so-called irreducible radius $r_{0}$ and irreducible mass $m_{0}$ through

$$
r_{0}=2 m_{0}=(A / 4 \pi)^{1 / 2} .
$$

This immediately implies that $a=r_{0}^{2} \Omega_{H}$. It turns out that the area is proportional to the thermodynamic entropy. Now imagine that we exchange some mass and some angular momentum with the hole, reversibly (and therefore at constant area) from just outside the horizon. These must be added according to

$$
d m=\Omega d(a m) .
$$

This equation can be integrated to give

$$
m=\frac{m_{0}}{\left[1-\left(\Omega r_{0}\right)^{2}\right]^{1 / 2}} .
$$

Imposing the condition $a<m$, we find that there is a mass $m-m_{0}<0.29 m$ that can, in principle, be extracted from the hole. The main way that this is thought to occur naturally is through the agency of large scale magnetic field that threads the event horizon of the black hole. This magnetic field can exert a torque on the hole, similar to the magnetic torques acting upon the sun and neutron stars, for example. Black hole spin provides a plausible power source for high energy phenomena like ultrarelativistic jets and gamma ray bursts and this is one reason why black holes are commonly thought to be spinning rapidly. (Even if the spin is not a significant power source, then the specific angular momentum of the gas that accretes onto a black hole is generally so large that it is very hard to imagine slowly spinning holes ever being formed.)

For present purposes, though, what is most important is that, in a rapidly spinning hole, the accreting matter can form a disk extending quite close to the horizon. Specifically, if we consider circular Keplerian orbits around a hole then these are stable down to a radius of marginal stability which is located at $6 \mathrm{~m}$ for a non-rotating (Schwarzschild) hole and approaches the horizon as $a \rightarrow m$. In addition, it is possible for strong pressure gradients within the disk to support matter in non-Keplerian orbits inside $6 \mathrm{~m}$. This means that gas may survive quite a long while in and around the ergosphere before crossing the horizon or being ejected. The dominant emission may come from this region, and as the radiation escapes, its trajectory and the propagation of its polarization can be significantly influenced by the curvature of the spacetime. This provides us with a potential probe of the Kerr metric. 


\subsubsection{Geometrical Optics of Plasma Waves in Flat Space}

We are interested in the propagation of plasma waves in the curved spacetime around a black hole. However, for the moment, let us consider the propagation of waves in flat space under geometrical optics. This is appropriate because the wavelengths that we are considering, at least for electromagnetic radiation, are always much smaller than the horizon radius. It is convenient to exploit the analogy with Hamiltonian particle dynamics. Under the eikonal approximation, we can define a phase $\phi$ such that $\nabla \phi=\vec{k}$ and $\partial \phi / \partial t=-\omega$. The phase velocity is, as usual, defined by $\vec{V}_{\phi}=\omega / \vec{k}$. We assume the existence of a dispersion relation

$$
\omega=\Omega(\vec{k}, \vec{x}, t) .
$$

Equivalently, there is a Hamilton-Jacobi equation

$$
\frac{\partial \phi}{\partial t}+\Omega(\nabla \phi, \vec{x}, t)=0
$$

which must be satisfied. The three Hamilton equations are

$$
\begin{aligned}
& \frac{d \vec{k}}{d t}=-\nabla \Omega, \\
& \frac{d \vec{x}}{d t}=\frac{\partial \omega}{\partial \vec{k}} \equiv \vec{V}_{g}, \\
& \frac{d \omega}{d t}=\frac{\partial \Omega}{\partial t},
\end{aligned}
$$

where

$$
\frac{d}{d t} \equiv \frac{\partial}{\partial t}+\vec{V}_{g} \cdot \nabla
$$

and $V_{g}$ is recognized as the group velocity. These three equations govern the propagation of plasma modes in a spatially inhomogeneous and temporally varying medium, under the short wavelength approximation. In a cold, unmagnetized plasma the dispersion relation is $\Omega=\left(\omega_{P}^{2}+c^{2} k^{2}\right)^{1 / 2}$ and $V_{g}=c^{2} / V_{\phi}$. We can think of wave quanta - plasmons - and the energy they carry, as moving along a path $\vec{x}(t)$ with the group velocity. These plasmons are conserved; i.e. the wave energy density $U$ can be shown to obey a conservation equation of the form

$$
\frac{\partial}{\partial t}\left(\frac{U}{\omega}\right)+\nabla \cdot\left(\frac{U \vec{V}_{g}}{\omega}\right)=0 .
$$

Consider, for example, shear Alfvén waves. The dispersion relation is $\omega=\vec{k} \cdot \vec{B} /(4 \pi \rho)^{1 / 2}$ and the group velocity is $\vec{V}_{g}=\vec{B} /(4 \pi \rho)^{1 / 2}$. The wave packets propagate along the magnetic field along with the energy although $\vec{k}$ can be directed at a large angle to $\vec{B}$.

The propagation of the polarization can be most simply approached by decomposing the given wave into its normal modes, propagating each along the direction of the group velocity, and compute the relative change in phase (to lowest order in the eikonal approximation this is simply $\int d x \cdot \vec{k}$ along the path.) Of course the character (i.e. polarization, local phase velocity etc. ) of these modes will change, but provided we are in the WKB limit, the modes are distinguished, non-degenerate (see below), and there is no mode crossing (which can occur and has to be handled more carefully), these changes will change adiabatically. As a result, they can be tracked and the total phase difference along a path can be computed.

The total flux can be computed by using the conservation of intensity along the path. Equivalently, we say that the phase space density of individual quanta of wave excitation, 
in individual modes is conserved along paths. If there is emission or absorption along the path then it is straightforward to write down the equation of radiative transfer and use the local emission and absorption coefficients to evolve the intensity (e.g. Rybicki \& Lightman 1979; Bekefi 1966).

In practice, of course, all of this can easily become quite involved. However it is important to understand the principles because these alert us to the sort of effects we might expect to observe.

\subsubsection{Magnetized Accretion Disk}

As a more pertinent illustration of some of these ideas, let us consider electromagnetic wave modes propagating through an accretion disk containing a strong, though disordered, magnetic field.

Consider a magnetoactive plasma with $X=\omega_{P}^{2} / \omega^{2}, Y=\omega_{G} / \omega<1$ where $\omega_{P}$ is the plasma frequency and $\omega_{G}$ is the electron gyro frequency. Under so-called "quasilongitudinal" conditions - essentially when $\cos \theta<Y$, where $\theta$ is the angle between $\vec{k}$ and $\vec{B}$, the electromagnetic eigenmodes are elliptically polarized with axis ratio $r=$ $1 \pm Y \sin \theta \tan \theta$ and phase velocity difference $\Delta V=c X Y \cos \theta$.

Now suppose that synchrotron (or cyclotron) radiation is emitted within an accretion disk of thickness $H$. The major axis of the polarization ellipse will be Faraday rotated at a rate $\Delta \Phi / d s=\Delta V \omega / 2 c^{2}$. Now if, as we expect, the magnetic field direction reverses often along a ray and if, as also anticipated, $X|Y| \omega H / c>>1$, then we expect that the emergent linear polarization will be vanishingly small (cf $\S 1.3 .4$ ). (The limiting polarization along an individual ray is likely to be determined by the decrease in the density rather than the magnetic field strength.)

The circular polarization is a bit more problematic. If we suppose that there is a net magnetic field normal to the disk, as is true of some models, then there should be a preferred sense of the circular polarization that is emitted in cyclotron or low energy synchrotron radiation. This will be largely preserved in propagating out of the disk.

If the emitted radiation is effectively unpolarized, we can analyze the production of circular polarization due to Faraday conversion by decomposing each wave into the eigenmodes. Thus consider a single eigenmode propagating out of the disk through a spatially varying magnetic field. We suppose that the variation happens relatively slowly on the scale of the wavelength so that the polarization ellipse adjusts adiabatically (with no mode crossings). Next, suppose that the two eigenmodes are launched with equal amplitude and that the field is uniform. The beating between the two eigenmodes will result in a circular polarization of amplitude $Y \sin \theta \tan \theta$ that changes sign as the plane of linear polarization rotates. If either the Faraday depth is large, or the sign of the magnetic field is as likely to be negative as positive, then the limiting circular polarization that emerges from the disk is equally likely to have either sign and so there will be no net circular polarization.

Now let the magnetic field direction vary along a ray. If the angle $\theta$ varies, then there will be a corresponding change in the axis ratio of the polarization ellipse, but still no preference for one sign over the other. However, if the azimuthal angle $\phi$ relating $\vec{B}$ to $\vec{k}$ changes in a systematic fashion along all rays then a net phase difference between the two modes will develop. In the same way that Faraday conversion creates circular polarization, such a phase difference will also create circular polarization. The main difference is that while Faraday conversion depends upon the direction of the magnetic field, and hence will not lead to a net polarization for randomized fields, the new mechanism depends upon the rate of shearing and only the strength of the field. Thus, it is possible to conceive of situations in which the rate of shearing and the typical length 
scales over which the magnetic field reverses are related in such a manner that a net circular polarization is produced without a commensurate linear polarization.

This situation is precisely what might be anticipated in a magnetized accretion disk. In the disk interior, the typical field direction will trail to reflect the differential rotation in the disk. However, the field will be swept back by progressively smaller angles as the ray approaches the disk surface, corresponding to a net rotation of the average azimuthal angle $\phi$. There will only be a preferred sense of limiting circular polarization, if the magnetostatic field is still changing in this systematic manner over the last radian of Faraday rotation. The net circular polarization will be $\sim c \ln Y / \omega H X$.

These are some of the subtle effects that could be present in an accretion disk and which could, under some circumstances, create measurable polarization, even in the absence of general relativity.

\subsubsection{Geometrical Optics of Vacuum Waves in a Curved Spacetime}

We first consider the propagation of photons in a vacuum surrounding a black hole. These follow orbits called null geodesics, just as material particles follow timelike geodesics. The equation of motion can be expressed in a general coordinate system though, in our case, Boyer-Lindquist coordinates, by saying that the total derivative of the wave vector along the ray vanishes. In index notation, this becomes

$$
k^{\beta} k_{; \beta}^{\alpha}=0,
$$

cf Eq. (5.62).

There are essentially three constants of the motion that describe these orbits, an energy, $-k_{0}$ an angular momentum, $k_{\phi}$, and a third quantity known as the Carter constant, $Q$ (e.g. Misner, Thorne \& Wheeler 1973.) (In fact, we only need the ratios $k_{\phi} / k_{0}, Q / k_{0}$ to define the rays.) Close to the black hole the rays are strongly curved with the consequence that a distant observer, able to resolve a black hole, would be able to see a distorted image of the disk behind the hole, apparently hovering above the hole (cf Fig. (2).). The ray trajectories are given, in general, by the solution of a set of coupled ordinary differential equations that can be partly integrated in terms of elliptic functions (Rauch \& Blandford, 1994). Given a model of the emission, for example of the surface emissivity of a thin accretion disk, it is a straightforward, though quite lengthy, exercise to compute the total emergent flux and, indeed, the form of the image that would be resolved if the black hole could be resolved.

Now, turn to the propagation of the polarization of vacuum modes in a curved spacetime, specifically outside the horizon of a Kerr hole (Laor, Netzer \& Piran 1990). As we have already emphasised, both non-thermal emission (e.g. synchrotron radiation) and electron scattering are likely to create polarized sources of radiation. If we just consider linear polarization for the moment, and the generalization to circular polarization is straightforward, then the question that we must answer is "How do we propagate the plane of polarization from one point to the next along a curving ray?". The answer is that the electric vector is "parallel-transported" (eg Misner, Thorne \& Wheeler 1973). What this means is that the unit vector in the direction of the electric field $\hat{e}^{\alpha}$ changes along the ray such that its magnitude and projection onto the direction of the ray remain constant. An additional constraint arising from Maxwell's equations in vacuum is that the electric vector must be perpendicular to the wave vector. Hence, in index notation, we have

$$
k^{\beta} \hat{e}_{; \beta}^{\alpha} ; \quad \hat{e}^{\alpha} k_{\alpha}=0 .
$$

These can be solved consistently to propagate the electric vector along a ray. 
What is actually done is somewhat different. It turns out that there is another conserved quantity, called the Walker-Penrose (1970) tensor, associated with the photon spinors (the familiar geometrical object that in this case are associated with light-like geodesics, the path taken by photons in vacuum.) This actually involves the electric vector and can be used to relate the polarization at the point of emission to that at the point of observation directly without having to integrate a differential equation (Connors, Stark \& Piran 1980). It is then possible to define a transfer function for the polarization and to compute the polarized flux given a specific emission model using the propagated intensity. (Note that, in propagating the intensity, we must correct for the Doppler and gravitational shifts. There is a natural way to do this in general relativity.)

\subsubsection{Geometrical Phase}

The next level of complication is to introduce the plasma into the curved space time. Let us do this in two stages. The first stage is to ignore the magnetic field so that the local dispersion relation takes the form $\Omega=\left(\omega_{p}^{2}(\vec{x})+c^{2} k^{2}\right)^{1 / 2}$. In this case, the refractive index is locally isotropic. This means that the two eigenmodes at a point are degenerate and that we have to formulate a rule to connect the polarization from one point along a path to the next.

The wave packets, which travel at the local group velocity, are no longer moving along null geodesics, but timelike geodesics instead. It turns out that the Hamiltonian equations of motion can be generalized in a covariant manner, provided that one has knowledge of the local linearized dispersion relation at every relevant point in space time. Therefore, there is a prescription for computing the paths. These can be described by a four velocity $u^{\alpha}$ and an acceleration $a^{\alpha}=d u^{\alpha} / d \tau$ with respect to a freely-falling frame, where $d \tau$ is an interval of proper time. The standard relativistic way to handle this is to generalize the notion of parallel-transport to Fermi-Walker transport (e.g. Misner, Thorne \& Wheeler 1973), which corrects for the non-null motion of the wave packets. The propagation equation becomes

$$
\frac{d \hat{e}^{\alpha}}{d \tau}=\hat{e}^{\beta} a_{\beta} u^{\alpha}-u_{\beta} \hat{e}^{\beta} a^{\alpha} .
$$

Eq. (5.69) reduces to Eq. (5.68) when Eq. (5.67) is satisfied. This provides a natural basis in which to discuss polarization propagation and phase changes.

It is instructive to consider a wave propagating along a twisting optical fiber, with $\vec{k}$ parallel to the local tangent to the fiber. Here again we have gradients in an isotropic refractive index. In this case, the unit electric vector, along $\hat{\vec{e}}$, must remain perpendicular

to the unit wave vector $\hat{\vec{k}}$. When the fiber bends, the change in $\hat{\vec{e}}$ must be along $\hat{\vec{k}}$; there is no other vector to be involved as the medium is isotropic. Therefore we can write down the equation of propagation for the electric vector from first principles.

$$
\frac{d \hat{\vec{e}}}{d s}=-\hat{\vec{k}}\left(\hat{\vec{e}} \cdot \frac{d \hat{\vec{k}}}{d s}\right) .
$$

This is a limiting case of Eq. (5.69).

A good way to visualize what is happening (Berry 1990) is to allow the tangent to the fiber to trace out a path on the unit sphere. $\hat{\vec{e}}$ is tangent to the sphere and it is straightforward to see that rotation angle of $\hat{\vec{e}}$ after traversing a complete circuit equals the solid angle enclosed by that circuit. If we propagate a linearly polarized wave along a twisting fiber, the polarization direction will, in general, be rotated between two points where the fiber is parallel. This experiment has been performed successfully (Chiao 
et al.1989). (Actually this was under conditions when physical as opposed to geometrical optics applies, though the results should be identical.)

This rotation - essentially a phase change between the two circularly polarized eigenmodes - is known as the geometric phase. Geometric phase is a quite general phenomenon in physics and analogs are expected to be relevant to wave propagation in a curved spacetime. The Foucault pendulum provides another example of this general phenomenon. As is well known, a Foucault pendulum at latitude $\ell$ will only rotate through an angle in inertial space of $2 \pi(1-\sin \ell)$, the solid angle traced out by the radial vector on the unit sphere, as it is carried around a complete circuit in one day by the spinning Earth. Now to see where general relativity may come in, it is helpful to consider a Foucault pendulum at the North pole. According to the above discussion, there is no rotation of the plane of oscillation according to Newtonian dynamics. However, the tiny dragging of inertial frames effect associated with the spin of the earth leads to an equally tiny rotation of the plane of polarization and there have been proposals to measure it. Effects such as these would be much larger near a spinning black hole and could also influence the propagation of electromagnetic waves.

\subsubsection{General Relativistic Magnetoionic Theory}

The second stage is to reinstate the magnetic field which, on general grounds, is surely present. This breaks the degeneracy between the two wave modes. This is akin to changing the pivot of a Foucault pendulum from a point attachment to an axle. As far as is known, there is no generalization of the Carter constant and the Walker-Penrose tensor, to non-null wave modes. The equations for the paths can be integrated and the intensity and the polarization can be propagated using the relativistic generalization of the approach outlined above (Broderick \& Blandford, in preparation.)

When a linearly-polarized vacuum wave crosses the ergosphere of a rapidly spinning hole, there is a contribution to the emergent polarization position angle of order unity due to the dragging of inertial frames. We can think of this as a phase difference in of order unity in the two circular polarized modes into which the linear mode can be decomposed. Now, if we introduce a magnetoactive plasma into the path, then there is likely to be a large Faraday rotation per unit length. The total rotation will differ by much more than $O(1)$ along different paths that any emitted linear polarization is likely to be erased. However, as discussed above, the eigenmodes are not completely circular and have an ellipticity $O(X Y)$. What this means is that a systematic phase difference $O(1)$ will be introduced between the two modes and that, if the original modes are in phase so that there is no circular polarization, a degree of polarization $O(X Y)$ will emerge, independent of the reversals of the magnetic field and variations in the total Faraday rotation along different lines of sight.

These matters deserve further attention.

\subsection{Interpretation}

\subsubsection{Sgr $A^{*}$ and other Low Luminosity AGN}

X-ray observations of Sgr A* (Baganoff et al.2001) have shown the the luminosity is very small $\left(\sim 4 \times 10^{33} \mathrm{erg} \mathrm{s}^{-1}\right)$ and the spectrum is quite soft. Variability on an hour timescale may also have been seen. (As has been argued elsewhere, Blandford \& Begelman 1999, this is generally to be expected if most of the mass supplied to the hole is blown away in a wind.) This suggests that the density of gas is very low close to the hole and opens up the possibility that we may be seeing radio or, more likely, mm emission from the ergosphere. Under these conditions, polarization observations could be quite diagnostic of the physical conditions. 
The variable circular polarization discussed above, increasing in degree with frequency, has at least three explanations. Firstly, there could be a radius to frequency mapping so that the radio photosphere shrinks with frequency and the field gets stronger so that the energy of the emitting relativistic electrons also decreases. This leads to an increase in the emitted degree of circular polarization (cf $\S 1$ ). Variability studies at high frequency should be quite diagnostic. Secondly, the polarization could be due to a flat space propagation effect along the lines discussed above. It will be particularly interesting to see if the sign of the circular polarization really does not change, as the observations to date may suggest. This could, in principle, be related to the angular velocity of the disk as outlined above, though quantitatively this seems improbable.

The third possibility may be the most unlikely, yet it is the most exciting. This is that the circular polarization reflect directly the geometry of the ergosphere and be due to a general relativistic, propagation effect as outlined above. In the case of $\mathrm{Sgr} \mathrm{A}^{*}$, we expect the gas at high latitude in the ergosphere to be moving with speed $\sim c$. We deduce that the expected degree of circular polarization is:

$$
C \sim X Y \sim 10^{-2}\left(\frac{\dot{M}}{10^{22} \mathrm{~g} \mathrm{~s}^{-1}}\right)\left(\frac{B}{100 \mathrm{G}}\right)\left(\frac{\lambda}{1 \mathrm{~cm}}\right)^{3} .
$$

It is not impossible that this effect is observable These matter deserve more attention, both observational and theoretical, in Sgr A* and other, nearby galaxies.

\subsubsection{Imaging the Ergosphere}

Interesting and timely as these ideas may be, radio observations are probably not likely to contribute to a confirmation of the essential features of the Kerr metric until we can actually image the ergosphere. At present, as we have remarked, the best resolution has been achieved in M87 $(\sim 100 m)$. Probably the best prospects lie with sub mm VLBI observations of Sgr A*, where interstellar scattering precludes resolving the ergosphere at radio wavelengths (Falcke, Melia \& Agol 1999). There are also quite futuristic plans to develop X-ray interferometry to achieve analogous goals (Cash et al.2000).

\subsection{Summary}

- Black holes are common features of the evolution of massive stars.

- Massive black holes are commonly found in the nuclei of normal galaxies. Presumably they powered active galactic nuclei including quasars and radio sources in the past.

- We have good grounds to be confident in the general theory of relativity and, specifically, the Kerr metric which describes the curved spacetime around a spinning black hole. However, this does not absolve us from the responsibility of testing the theory.

- We also want to understand how black holes accrete and how they form jets as well as their impact on Galactic and extragalactic astronomy.

- Recent observations suggest that we may be observing radio and mm emission from very close to the black hole in Sgr A*. There is consequently interest in developing the magnetoionic theory and radiative transfer in a general relativistic environment.

RB thanks Javier Trujillo Bueno and the Director of the Instituto de Astrofisica de Canarias for their hospitality, his fellow lecturers for their instruction and the students for their attention and questions. The NSF and NASA are acknowleged for support under grants AST 99-00866 and 5-2837 respectively. 


\section{REFERENCES}

DISKS

Agol, E. \& Blaes, O. M. 1996, MNRAS, 282, 965

Angel, J. R. P., 1969, ApJ 158, 219

Chandrasekhar, S., 1960, Radiative Transfer, New York, Dover

Chen, K. \& Eardley, D. M. 1991, ApJ, 382, 125

Connors, P. A., Stark, R. F., \& Piran, T. 1980, ApJ, 235, 224

Draine, B. T. \& Lee, H. M. 1984 ApJ, 285, 89

Fabian, A. C., Iwasawa, K., Reynolds, C. S., \& Young, A. J. 2000, PASP, 112, 1145

Frank, J., King, A., \& Raine, D. 1992, Accretion Power in Astrophysics, Cambridge University Press, Cambridge

Gierlinski, M., Zdziarski, A. A., Poutanen, J., Coppi, P. S., Ebisawa, K., \& Johnson, W. N. 1999, MNRAS, 309, 496

Hubeny, I., Agol, E., Blaes, O., \& Krolik, J. H., 2000, ApJ, 533, 710

KÖnigl, A. \& KartJe, J. F. 1994, ApJ, 434, 446

Koratkar, A. \& Blaes, O. M. 1999, PASP, 111, 1

Krolik, J., 1999, Active Galactic Nuclei: from the Central Black Hole to the Galactic Environment, Princeton University Press, Princeton

Laor, A., Netzer, H., \& Piran, T. 1990, MNRAS, 242, 560

Matt, G., Fabian, A. C., \& Ross, R. R., 1993, MNRAS, 264, 839

Meszaros, P., Novick, R., Szentgyorgyi, A., Chanan, G. A., Weisskopf, M. C., 1988, ApJ, 324, 1056

Nandra, K., George, I. M., Mushotzky, R. F., Turner, T. J., \& Yaqoob, T., 1999, ApJ, 523, L17

Poutanen, J. \& Svensson, R., 1996, ApJ, 470, 249

Pringle, J., 1997, MNRAS, 292, 136

Shapiro, S. L. \& Teukolsky, S. A., 1983, Black Holes, White Dwarfs, and Neutron Stars, Wiley-Interscience, New York

Young, A. J. \& Reynolds, C. S., 2000, ApJ, 529, 101

Zubko, V. G. \& Laor, A., 2000, ApJS, 128, 245

JETS

Akeson, R. \& Carlstrom, J., 1999, ApJ, 491, 254

Blandford, R. D. \& Königl, A., 1979, ApJ 232, 34

Blandford, R. D., Netzer, H., Woltjer, L., Courvoisier, T. J. -. \& Mayor, M. 1990, Saas-Fee Advanced Course 20. Lecture Notes 1990. Swiss Society for Astrophysics and Astronomy, XII, Springer-Verlag Berlin Heidelberg New York

Bower, G., 2000, GCNEWS - Galactic Center Newsletter, vol. 11, p. 4-6 (eds. A. Cotera, H. Falcke, \& S. Markoff)

Helfand, D. J., Gotthelf, E. V. \& Halpern, J. P., 2001, ApJ, in press

Jones, T. W. \& O’Dell, S. L., 1977, ApJ 214, 522

Kellermann, K. I. \& Pauliny-Toth, I. I. K., 1969, ApJL, 155, L71

Koopmans, L. V. E. \& De Bruyn, A. G., 2000, A\&A 358, 793

Macquart, J.-P. \& Melrose, D. B., 2000, ApJ accepted, astro-ph/0007429

Mirabel, I. F. \& Rodriguez, L. F., 1994, Nature 371, 46

Perlman, E. S., Biretta, J. A., Zhou, F., Sparks, W. B. \& Macchetto, F. D., 1999, AJ 117,2185

Reynolds, C. S., Fabian, A. C., Celotti, A. \& Rees, M. J., 1996, MnRas

Rybicki, G. B. \& Lightman, A. P., 1979, New York, Wiley-Interscience 
Wardle, J. F. C., Homan, D. C., Ojha, R. \& Roberts, D. H., 1998, Nature 395, 457

Weisskopf, M. et al., 2000, ApJ, 536, L81

Wilson, A. S., Young, A. J. \& Shopbell, P. L., 2001, ApJ 546, in press, (astro-ph/0008467) OUTFLOWS

Antonucci, R., 1993, ARAA, 31, 473

Arav, N., Korista, K. T., Barlow, T. A. \& Begelman, M. C., 1995, Nature, 376, 576

Cohen, M. H. et al., 1995, ApJ, 448, L77

Koratkar, A., Antonucci, R. R. J., Goodrich, R. W., Bushouse, H. \& Kinney, A. L., 1995, ApJ, 450, 501

Koratkar, A., Antonucci, R. R. J., Goodrich, R. W., And Storrs, A., 1998, ApJ, 503, 599

Korista, K. \& Ferland, G., 1998, ApJ, 495, 672

LEe, H. -W., 1994, MNRAS, 268, 49

LeE, H. -W. \& Blandford, R. D., 1997, MNRAS, 288, 19

Murray, N., Chiang, J., Grossman, S. \& Voit, M., 1995, ApJ, 451, 498

Ogle, P. M., Cohen, M. H., Miller, J. S., Tran, H. D., Goodrich, R. W. \& Martel, A. R.. 1999, ApJS, 125, 1

Rybicki, G. B. \& Hummer, D. G., 1978, ApJ, 219, 654

Weymann, R. J., Morris, S. L., Foltz, C. B., \& Hewett, P. C., 1991, ApJ, 373, 23

Koratkar, A., Antonucci, R. R. J., Goodrich, R. W., Bushouse, H., \& Kinney, A. L., 1995, ApJ, 450, 501

Lee, H. -W. \& Ahn, S. -H., 1998, ApJ, 504, L61

Lee, H. -W. \& Blandford, R. D., 2000, ApJ, submitted

Nussbaumer H., Schmid, H. M., \& Vogel, M., 1989, A\& Ap, 211, L27

Stenflo, J. O., 1980, A\& Ap, 84, 68

NEUTRON STARS

Adler, S. L., 1971, Ann. Phys., 67, 599.

Arons, J. \& Barnard, J. J., 1986, ApJ, 302, 120

Baring, M. G. \& Harding, A. K. 1997, ApJ, 482, 372.

BeCker, W., 2000, Adv. Sp. Res., 25, 647

Chakrabarty, D. \& Kaspi, V., 1998, ApJ, 498, L37

Cheng, A. F. \& Ruderman, M. A. 1979, ApJ, 229, 348.

Cordes, J. M., 2001, ApJ, inpress

Daugherty, J. K. \& Harding, A. K. 1982, ApJ, 252, 337.

Daugherty, J. K. \& Harding, A. K. 1983, ApJ, 273, 761.

Gwinn, C. R., 2000, ApJ, 531, 902

Heyl, J. S. \& Hernquist, L., 1997a, Journ. Phys. A, 30, 6485.

Heyl, J. S. \& Hernquist, L., 1997b, Phys. Rev. D, 55, 2449.

Heyl, J. S. \& Shaviv, N., 2000, Mon. Not. Royal Astr. Soc., 311, 555.

Heyl, J. S. \&, Shaviv, N., 2001, Nature, submitted.

Hirano, C. \& Gwinn, C. R., 2001, ApJ, in press

Lodenqual, J., Canuto, V., Ruderman, M. \& Tsuruta, S., 1974, ApJ 190, 141.

Lyne, A. G. \& Smith, F. G., 1998, Pulsar Astronomy, Cambridge: Cambridge University Press

Lyutikov, M., 1998, MNRAS, 298, 1198

Lyutikov, M., Blandford, R. \& Machabeli, G., 1999, MnRAS, 305, 338

MÉsz'Aros, P., 1992. High Energy Radiation from Magnetized Neutron Stars, Chicago: Uni- 
versity of Chicago Press

Pavlov, G. G \& Shibanov, I. A., 1978, AZh, 55, 373.

Pavlov, G. G \& Zavlin, V. E., 2000, ApJ, 529, 1011.

Shaviv, N. J., Heyl, J. S. \& Lithwick, Y., 1999, Mon. Not. Royal Astr. Soc., 306, 333.

Thompson, A. C. \& Duncan, R., 1995, MnRAS, 275, 255

Thompson, D., 2000, Proc. International Symposium on High Energy Astrophysics, Heidelberg, (in press) astro-ph/0101039

Usov, V. V. \& Melrose, D. B., 1996, ApJ, 464, 306.

BLACK HOLES

Baganoff, F. et al., 2001, ApJ, submitted

Bekefi, G., 1966, Radiation Processes in Plasmas, New York: Wiley

Berry, M. V., 1990, Phys. Today, 12, 34

Blandford, R. D. \& Begelman, M. C., 1999, MNRAS, 303, L1

Bower, G. C., Falcke, H. \& Backer, D. C., 2000, ApJ, 523, L29

Bower, G. C., Wright, M. C. H., Backer, D. C. \& Falcke, H., 2000, ApJ, 527, 851

Cash, W., Shipley, A., Osterman, S. \& Joy, M., 2000, Nature, 407, 160

Chino, R. Y., Tomita, A. \& Wu, Y-S, 1989, in Geometric Phases in Physics, ed. A. Shapere \& F. Wilczek, Singapore: World Scientific

Falcke, H, Melia, F., \& Agol, E., 1999, ApJ 528, L13

Ghez, A., Morris, M., Becklin, E. E., Kremenek, T. \& Tanner, A., 2000, Nature, 407,349

LaOr, A., Netzer, H. \& Piran, T., 1990, MNRAS, 242, 560

Lo, K.-Y., Shen, Z.-Q., Zhao, J.-H. \& Ho, P. T. P., 1999, The Central Parsecs, ed. Falcke et al., Berkeley:ASP

Misner, C, Thorne, K. S. \& Wheeler, J. A., 1973, Gravitation, San Francisco: Freeman

Rauch, K. P. \& Blandford, R. D., 1994, ApJ, 421, 46

Walker, M. \& Penrose, R., 1970, Comm. Math. Phys., 18, 265

Zhao, J.-H., Bower, G. C. \& Goss, W. M., 2001, ApJ, in press 